\title{
ANÁLISIS FLORÍSTICO DE DOS ÁREAS CON BOSQUE MESÓFILO DE MONTAÑA EN EL ESTADO DE HIDALGO, MÉXICO: ELOXOCHITLÁN Y TLAHUELOMPA ${ }^{1}$
}

\author{
Othón Alcántara Ayala \\ E \\ ISOLDA LUNA VEGA \\ Herbario FCME, Departamento de Biología \\ Facultad de Ciencias \\ Universidad Nacional Autónoma de México \\ Ciudad Universitaria, Apartado Postal 70-399 \\ 04510 México, D.F.
}

\section{RESUMEN}

Los bosques mesófilos de Eloxochitlán y Tlahuelompa, enclavados en la Sierra Madre Oriental y en la Huasteca Hidalguense, representan dos manchones con diferente composición florística en el estado de Hidalgo. Estos bosques en general presentan tres estratos arbóreos: uno alto (20-30 m y hasta $40 \mathrm{~m})$, uno medio $(8-20 \mathrm{~m})$ y uno bajo $(2-8 \mathrm{~m})$. Se ofrece una lista de plantas vasculares de ambos sitios constituida por 394 especies, 266 géneros y 110 familias. Al realizar un análisis de la distribución actual de los géneros, se observa que gran parte de ellos se conocen exclusivamente del continente americano (93 géneros, 35\%) y sus especies generalmente son componentes del sotobosque; la mayoría de los géneros del estrato arbóreo se distribuyen principalmente en el hemisferio norte (25 géneros, 9.4\%). Los rodales hidalguenses de Fagus grandifolia ssp. mexicana se proponen como áreas de reserva, dado el carácter relictual y endémico de este taxon.

Palabras clave: florística, bosque mesófilo de montaña, Eloxochitlán, Tlahuelompa, estado de Hidalgo, México, distribución.

\section{ABSTRACT}

The cloud forests of Eloxochitlán and Tlahuelompa represent two fragments with different floristic composition of the Hidalgo State, México, in the Huasteca region of the Sierra Madre Oriental. In the arboreal vegetation three vertical strata can be distinguished: high (20-30 or even $40 \mathrm{~m}$ ), medium (8$20 \mathrm{~m}$ ), and lower $(2-8 \mathrm{~m})$. A floristic list of vascular plants of both areas constituted by 394 species, 266 genera, and 110 families is presented. An analysis of the current distribution of the genera reveals that many of the genera are distributed exclusively in America, usually those of the understory (93 genera, $35 \%$ ); most genera of the canopy trees are distributed mainly in the Northern Hemisphere (25 genera, 9.4\%). The areas with Fagus grandifolia ssp. mexicana in the state of Hidalgo are suggested as reserve areas due to the relictual and endemic character of this taxon.

1 Trabajo realizado con apoyo económico del Consejo Nacional de Ciencia y Tecnología 31879-N y parcialmente por el proyecto $\mathrm{H} 102$ de la Comisión Nacional para el Conocimiento y Uso de la Biodiversidad. 
distribution.

Key words: floristics, cloud forest, Eloxochitlán, Tlahuelompa, Hidalgo State, Mexico,

\section{INTRODUCCIÓN}

Hidalgo es el tercer estado de la República Mexicana con mayor superficie ocupada por bosque mesófilo de montaña, después de Oaxaca y Chiapas (Ortega y Castillo, 1996). Este tipo de vegetación ha sido catalogado como uno de los que posee mayor riqueza por unidad de superficie en el país (Rzedowski, 1996); su distribución fragmentaria a lo largo de las principales sierras, así como la compleja relación geográfica de sus elementos florísticos hacen que esta comunidad sea muy interesante desde una perspectiva biogeográfica.

De acuerdo con Rzedowski (1996), la riqueza de endemismos a nivel de género en este tipo de vegetación es escasa y poco se sabe realmente de su cuantía a nivel de especie, aunque se considera que es relativamente alta, particularmente en lo que concierne a las plantas arbóreas. La carencia en este conocimiento hace necesario intensificar los estudios regionales que permitan conocer las particularidades florísticas de cada área.

Se ha postulado que el bosque mesófilo mexicano tiene una relación fitogeográfica estrecha con los bosques caducifolios del este de Estados Unidos y con el este de Asia (Sharp, 1953, 1966; Matuda, 1953; Sharp e Iwatsuki, 1965; Puig, 1989); más recientemente se ha insistido en los fuertes vínculos de la flora de este tipo de vegetación con bosques similares de Sudamérica, en particular con los de la región andina (Rzedowski, 1996). Todas estas consideraciones, sin embargo, han sido propuestas dentro de un contexto dispersalista, donde México es considerado como receptor de ciertos elementos florísticos y como centro de origen y radiación de otros (Rzedowski, 1996), siendo transicional entre las regiones Neártica y Neotropical. Actualmente se han venido utilizando enfoques vicariancistas que sustituyen al dispersalista, como los empleados por Rosen (1978) para peces de agua dulce, Liebherr (1991) para coleópteros y Luna et al. (1999) y Luna et al. (2000) para plantas vasculares.

El estudio del patrón fragmentario y la obtención de listas florísticas completas de los distintos manchones con este tipo de vegetación en el país, nos han permitido establecer la relación que existe entre cada una de las áreas inventariadas (Luna et al., 1999), por ejemplo cuál es su vinculación florística, y con ello contrastar la regionalización biológica que varios autores han hecho del país (por ejemplo Rzedowski, 1978 y Morrone et al., 1999, entre muchos otros). De igual manera, han permitido proponer hipótesis sobre los procesos que han producido los patrones actuales de distribución de la flora y fauna en México.

Los inventarios florísticos regionales y bióticos en general, son herramientas primordiales en el establecimiento de programas adecuados de conservación. De acuerdo con Humphries et al. (1991), para determinar regiones prioritarias a conservar es preciso contar con listados que contengan la mayor diversidad de especies en un mínimo de áreas; para ello, una vez elegida la primera área, las subsiguientes deben ser seleccionadas de manera que incrementen el número de taxa conservados al máximo posible. Para otros autores sería más importante el establecimiento de reservas en función de la abundancia de elementos endémicos (Williams y Humphries, 1994). 
Las metas del presente trabajo consisten en contribuir al conocimiento de la riqueza vegetal de los bosques montanos de la Huasteca Hidalguense, en particular dar a conocer la composición florística del bosque mesófilo de montaña de dos manchones con este tipo de vegetación ubicados en las cercanías de Eloxochitlán y de Tlahuelompa, así como brindar las bases para proponer los rodales de Fagus grandifolia ssp. mexicana como áreas de reserva de este árbol dado el carácter relictual y endémico del mismo.

\section{ANTECEDENTES}

Miranda y Sharp (1950) describieron someramente las distintas asociaciones vegetales de las regiones de Zacualtipán, San Bartolo Tutotepec y Chapulhuacán, Hidalgo. Puig (1976) efectuó estudios en varias localidades del estado, como Chapulhuacán, Ixtlahuaco, Tlanchinol, Xochicoatlán, Tianguistengo, Zacualtipán, Tenango de Doria, Xochiatipán, Tutotepec y Acaxochitlán, considerando a sus comunidades boscosas como parte de sus formaciones tropicales de altitud; este autor ofrece un listado florístico para Tlahuelompa constituido por 52 especies de plantas vasculares. Ehnis (1981) estudió la ecología e importancia de los bosques de hayas (Fagus mexicana) de Teziutlán, Puebla y Zacualtipán, Hidalgo y consideró conveniente establecer medidas para la protección de esta especie. Pérez (1994) realizó un estudio dendrológico y silvícola del género Fagus para los estados de Tamaulipas, Hidalgo y Puebla y concluyó que Fagus grandifolia ssp. mexicana es una entidad amenazada, ya que el rodal más importante de este árbol, ubicado en los alrededores de Tlahuelompa, Hidalgo, sólo contiene aproximadamente 10,000 individuos. Luna et al. (1994), al igual que Alcántara y Luna (1997) realizaron estudios florísticos del bosque mesófilo de montaña de los municipios de Tlanchinol y Tenango de Doria respectivamente, así como un análisis de la distribución actual de las familias y géneros de plantas vasculares; finalmente Mayorga et al. (1998) publicaron trabajos similares para el bosque de Molocotlán de los municipios de Molango y Xochicoatlán, Hidalgo.

\section{ÁREA DE ESTUDIO}

Localización y ruta de acceso

La región de estudio pertenece al municipio de Eloxochitlán se localiza aproximadamente entre los $20^{\circ} 43^{\prime}$ y los $20^{\circ} 46^{\prime}$ de latitud norte y los $98^{\circ} 45^{\prime}$ y $98^{\circ} 47^{\prime}$ de longitud oeste (Fig. 1), y queda situada dentro de la Sierra Madre Oriental.

A esta área se llega por la carretera federal 85 México-Pachuca, la que se continúa por la 105 Pachuca-Tampico; aproximadamente en el kilómetro 124, se inicia un camino de terracería que conduce al poblado de San Agustín Eloxochitlán. El fragmento de bosque se halla entre los kilómetros cuatro y nueve de este camino.

Tlahuelompa está ubicado en el municipio de Zacualtipán, el cual pertenece a la región de Molango, integrada por once municipios. El área de estudio de esta zona se encuentra entre los $20^{\circ} 36^{\prime}$ y $20^{\circ} 38^{\prime}$ de latitud norte y los $98^{\circ} 34^{\prime}$ y los $98^{\circ} 38^{\prime}$ de longitud oeste (Fig. 1). 

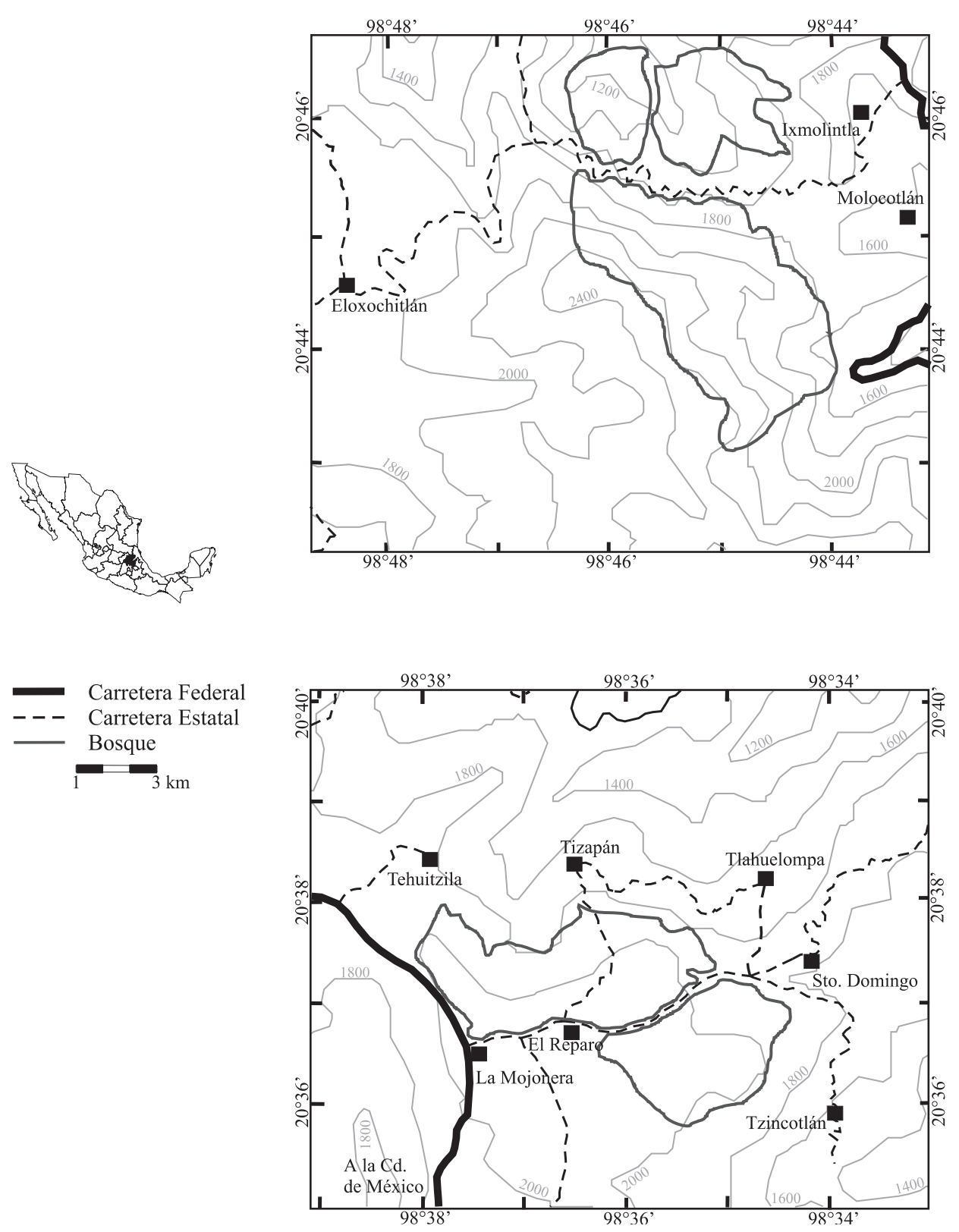

Fig. 1. Localización de las áreas de estudio. 
A esta zona de Tlahuelompa también se llega por las carreteras federales 85 MéxicoPachuca y 105 Pachuca-Tampico; esta última entronca con la carretera ZacualtipánTlahuelompa. Aproximadamente en el kilómetro seis de ésta se localiza la entrada al manchón de bosque.

\section{Clima}

De acuerdo con el mapa publicado por la Comisión Nacional para el Conocimiento y Uso de la Biodiversidad (Anónimo, 1997) en el área de estudio próxima a Eloxochitlán predomina un clima templado subhúmedo $\mathrm{C}\left(\mathrm{w}^{2}\right) \mathrm{w}\left(\mathrm{i}^{\prime}\right) \mathrm{gw}$ ", con poca oscilación térmica, oscilación anual de las temperaturas mensuales entre 5 y $7^{\circ} \mathrm{C}$, marcha de temperatura tipo Ganges, con canícula, temperatura media anual de $16.5^{\circ} \mathrm{C}$, precipitación media anual de $1075 \mathrm{~mm}$ y periodo de lluvias de junio a octubre. No se cuenta con la información necesaria para elaborar el diagrama ombrotérmico.

La estación Zacualtipán, ubicada muy cerca de Tlahuelompa, tiene un clima semifrío, húmedo, Ccm(f)(e)gw", con régimen de lluvias intermedio, marcha de temperatura tipo Ganges y presencia de canícula, temperatura media anual de $11.2^{\circ} \mathrm{C}$, precipitación anual de 1948.9 mm (García, 1988) (Fig. 2). No obstante, Pérez (1999) sostiene que el clima de Montes de Zacatlamaya, Hidalgo corresponde al tipo $\mathrm{Cbm}(\mathrm{f})(\mathrm{e}) \mathrm{gw}$ ", algo diferente al de la estación Zacualtipán y con clima templado.

Fisiografía

Ambas localidades se encuentran en la parte meridional de la Sierra Madre Oriental, que se caracteriza por presentar sierras con una orientación noroeste-sureste, constituidas por rocas paleozoicas y mesozoicas. Éstas se encuentran intrusionadas por rocas ígneas de composición granodiorítica del Terciario (Anónimo, 1992b). De igual manera ambas zonas pertenecen a la subprovincia del Carso Huasteco, dominada por sierras que constituyen la región conocida como Huasteca Hidalguense, en los sistemas de topoformas clasificados como sierras de laderas abruptas, en su mayoría de origen sedimentario y litología de calizas y areniscas-conglomerado.

La variación altitudinal en la zona de Eloxochitlán va principalmente de los 1400 a los 2210 m s.n.m, aunque existen algunas áreas de mayor altitud, como es el caso del cerro Agua Fría el cual alcanza los 2600 m s.n.m., y barrancas muy profundas como las de Rincón Axotla, barranca Honda y la barranca Agua Fría, lo cual le confiere un relieve muy accidentado (Anónimo, 1989).

En el área de Tlahuelompa la topografía también es muy abrupta con pendientes muy pronunciadas y escarpadas; aquí la variación altitudinal va de los 1780 a los 2000 m s.n.m. existiendo sólo pocos lugares planos en los márgenes de los arroyos (Anónimo, 1991). Las pendientes son mucho más pronunciadas en la porción norte, llegando a ser de más de $100 \%$ (Ehnis, 1981). 


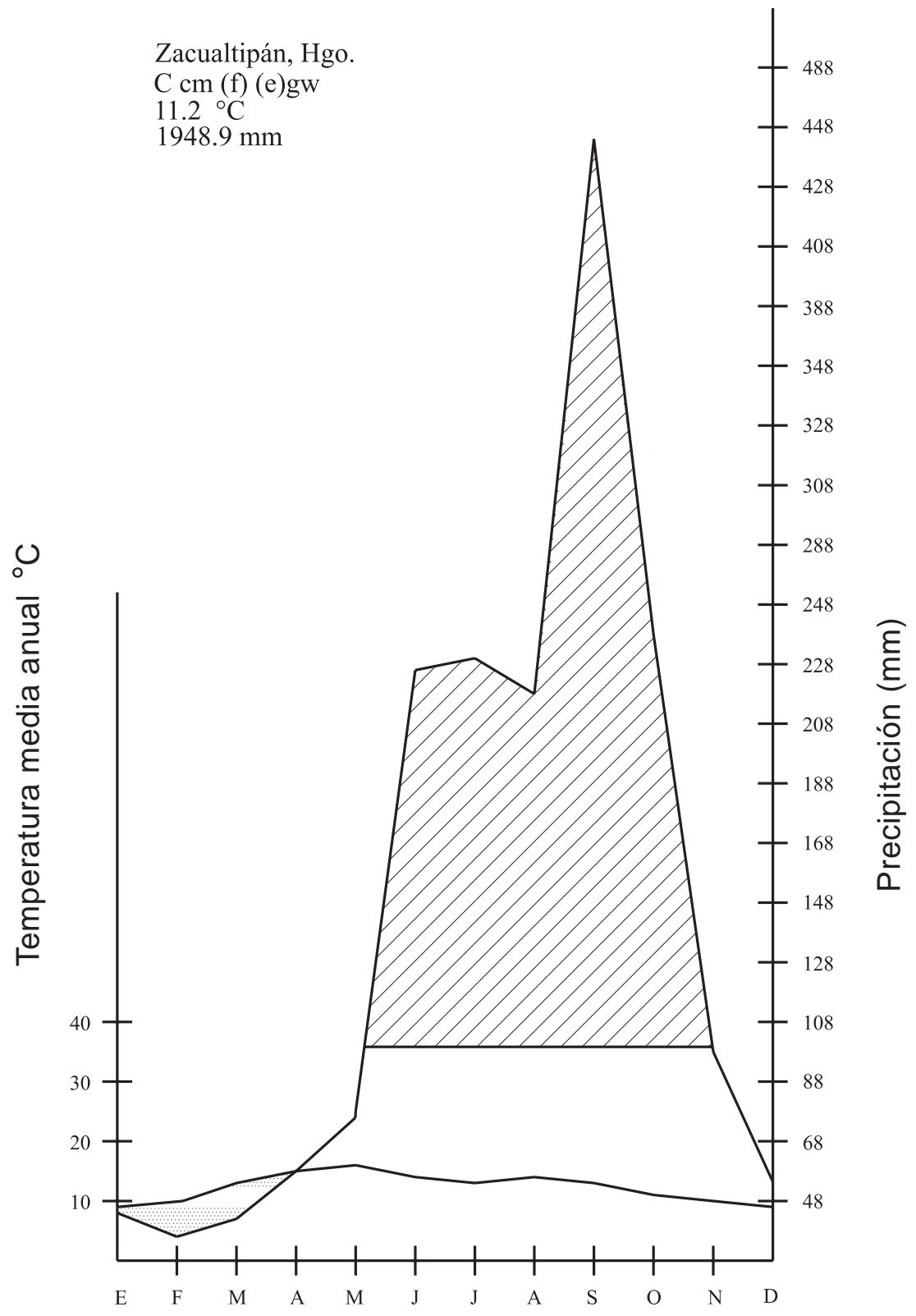

Fig. 2. Diagrama ombrotérmico de la estación Zacualtipán, Hidalgo. 
Alcántara y Luna: Análisis Florístico de dos Áreas con Bosque Mesófilo de Montaña

Geología

Estructuralmente ambas zonas pertenecen al anticlinorio de Huayacocotla que forma parte del cinturón de pliegues y cabalgaduras de la Sierra Madre Oriental. En términos de geología económica a esta zona se le conoce como Distrito Manganesífero de Molango (Ochoa-Camarillo, 1997).

En Eloxochitlán se distinguen tres estratos geológicos principales: 1) hacia el noreste predomina un estrato del Jurásico Superior, formado de rocas sedimentarias y vulcanosedimentarias de caliza-lutita, 2) hacia el este sobresale un estrato del Cretácico Inferior formado de rocas calizas (Anónimo, 1983) y 3) algunas mesas formadas por derrames basálticos miocénicos, que ocultan parcialmente las estructuras preexistentes. Los basaltos se encuentran en las partes más elevadas, generalmente por arriba de los 1000 m s.n.m. y sus límites muestran una topografía muy abrupta. Estas mesas basálticas están surcadas por barrancas muy profundas que generalmente forman acantilados (Ochoa-Camarillo, 1997).

En el área de Tlahuelompa se pueden distinguir dos estratos geológicos; el primero corresponde al Terciario Superior (en los poblados de Tehuitzila, Tlahuelompa, Zietla y alrededores), formado principalmente de rocas ígneas extrusivas del tipo de tobas ácidas y un segundo que corresponde al Jurásico Inferior (poblados El Reparo, Santo Domingo, Tzincotlán y alrededores), constituido mayormente de rocas sedimentarias de lutita-arenisca. Las rocas que se observan en la región son de origen volcánico; abunda en gran parte la obsidiana (Anónimo, 1983).

Influencia humana

Gran parte de las actividades agrícolas que se practican en el municipio de Eloxochitlán están enfocadas a la siembra de maíz y frijol y en mucha menor proporción a la de calabaza, naranja y manzana, aunque el uso del suelo es primordialmente de agostadero (en la cría y explotación bovina y porcina), le sigue el agrícola y por último el forestal. La mayor parte de la superficie es de pequeña propiedad y ejidal (Anónimo, 1996). Estas actividades se realizan a expensas del bosque, ya que cada vez es más notoria la disminución de éste y la expansión de las áreas dedicadas a la siembra de cultivos y la cría de ganado.

En Tlahuelompa se fabrican campanas de bronce y se usa carbón para la fundición del metal; parte de este carbón es suministrado por ejidatarios de "La Mojonera", que lo obtienen de Pinus patula, aplicando técnicas tradicionales del centro del país, que han sido heredadas de una generación a otra. El principal uso del suelo es el forestal $(65.5 \%)$, de agostadero (13.8\%) y para otros fines (12.2\%). En cuanto a la tenencia de la tierra $72.2 \%$ es pequeña propiedad y $27.8 \%$ es ejidal (Pérez, 1994).

Los montes de Zacatlamaya, cerca de Zacualtipán, lugar donde se localiza el bosque de Fagus, está en posesión de 84 ejidatarios de "La Mojonera". En los alrededores se desarrollan actividades agrícolas, para lo que se desmonta el bosque con el propósito de sembrar maíz, frijol y calabaza. También se producen frutales como manzana y pera, con problemas de parasitismo por muérdago y se explota la madera de Quercus spp., Clethra 
spp. y de Pinus patula para construcción. El bosque de Fagus no se explota desde 1940, año en el que se impuso la veda; sin embargo existe interés por iniciar nuevamente esta actividad por parte de los ejidatarios (Pérez, 1994).

\section{MÉTODOS}

Se realizaron doce visitas al área de estudio en diferentes épocas del año, de marzo de 1995 a noviembre de 1996; los sitios de recolecta se eligieron a través de una prospección de los municipios con base en mapas (carreteras 1: 400 000, topográfico 1:50 000) y fotos aéreas (1:75 000), procurando que fueran los lugares con vegetación mejor conservada.

Durante las visitas se realizó la recolección de muestras de especies de plantas vasculares en estado de floración y/o fructificación y se hicieron observaciones que permitieron describir cualitativamente a estas comunidades. Asimismo se elaboraron los perfiles esquemáticos de los bosques de estas zonas (Fig. 3).

Los ejemplares obtenidos se determinaron en lo posible hasta el nivel de especie, la mayoría de ellos se depositaron en el Herbario de la Facultad de Ciencias (FCME). En algunos casos se contó con la ayuda de especialistas.

Se obtuvo una lista florística de plantas vasculares recolectadas en ambas zonas, agrupando alfabéticamente tanto a los géneros como a las familias; para las angiospermas y gimnospermas se utilizó el sistema de Engler y Diels (1936) y para las pteridofitas y grupos afines el propuesto por Mickel y Beitel (1988). Los autores de las especies se abreviaron de acuerdo con Brummitt y Powell (1992). La distribución actual de los géneros se obtuvo a partir de los trabajos de Willis (1973) y Mabberley (1997), agrupándose a éstos en seis categorías.

\section{RESULTADOS}

Análisis florístico

Hasta el momento se cuenta con una lista florística de ambas áreas compuesta por 394 especies de plantas vasculares, pertenecientes a 266 géneros y 110 familias (Apéndice). Las dicotiledóneas son el grupo de plantas más numeroso, seguido por las monocotiledóneas, pteridofitas y gimnospermas (Cuadro 1).

Las familias más importantes en cuanto a número de especies para ambas localidades son Compositae (con 30 especies para Eloxochitlán y 24 para Tlahuelompa) y Solanaceae (con 15 para Eloxochitlán y 13 para Tlahuelompa); en Eloxochitlán otras familias bien representadas son Labiatae (12), Leguminosae, Orchidaceae (con 10 cada una), Rubiaceae (9), Fagaceae, Rosaceae (con 7 cada una), Aspleniaceae, Begoniaceae y Urticaceae (con 6 cada una); en Tlahuelompa, en cambio, están bien representadas las Fagaceae, Rubiaceae (con 10 especies cada una), Labiatae, Orchidaceae, Rosaceae (con 8 cada una) y Ericaceae (6). Los géneros con mayor número de especies para Eloxochitlán son Salvia (10), Quercus, Solanum (con 7 cada uno), Begonia (6), Ageratina, Polypodium 


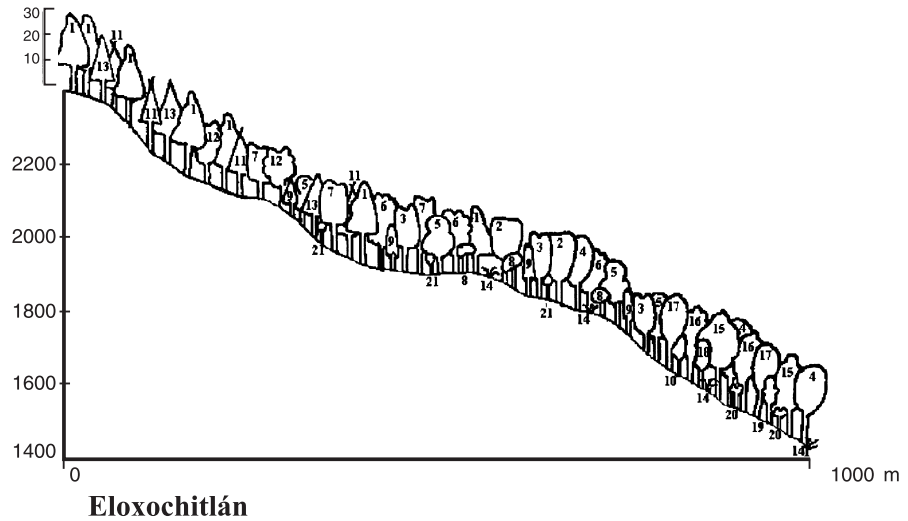

$\begin{array}{ll}\text { 1. Cupressus benthamii } & \text { 12. Quercus crassifolia } \\ \text { 2. Carpinus caroliniana } & 13 . \text { Pinus patula } \\ \text { 3. Quercus sartorii } & 14 . \text { Ceratozamia mexicana } \\ \text { 4. Tilia houghii } & 15 . \text { Meliosma alba } \\ \text { 5. Magnolia schiedeana } & 16 . \text { Persea americana } \\ \text { 6. Quercus germana } & 17 . \text { Quercus acutifolia } \\ \text { 7. Quercus affinis } & 18 . \text { Clethra alcoceri } \\ \text { 8. Bauhinia chapulhuacania } & 19 . \text { Ocotea klotzschiana } \\ \text { 9. Clethra mexicana } & \text { 20. Perrottetia ovata } \\ \text { 10. Styrax glabrescens } & 21 . \text { Rhamnus pompana } \\ \text { 11. Pinus pseudostrobus } & \end{array}$

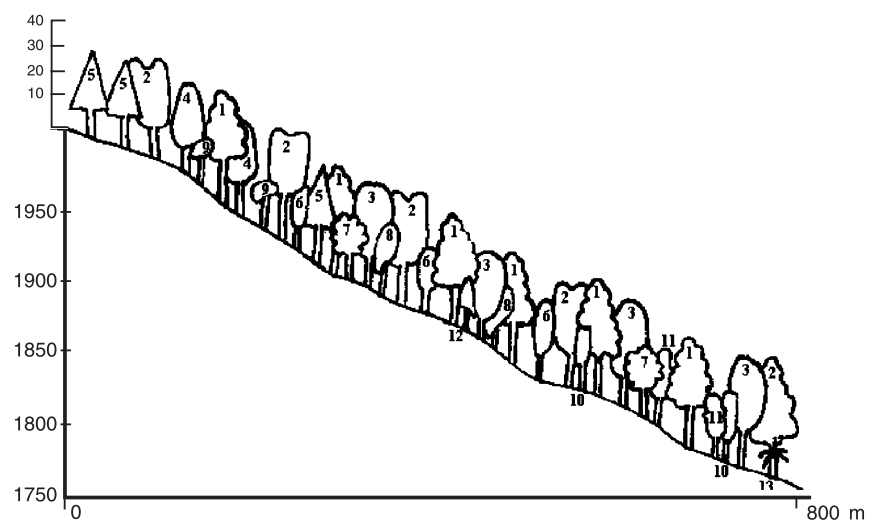

\section{Tlahuelompa}

1. Fagus grandifolia ssp. mexicana

2. Quercus sartorii

8. Ostrya virginiana

3. Quercus trinitatis

4. Quercus eugeniifolia

Cleyera theaeoides

5. Pinus patula

6. Clethra mexicana

7. Magnolia schiedeana

11. Ocotea klotzschiana

12. Ternstroemia huasteca

13. Cyathea fulva

Fig. 3. Perfiles esquemáticos de la vegetación de Eloxochitlán y Tlahuelompa, Hidalgo. 
y Smilax (con 4 cada uno); para Tlahuelompa son Quercus (9), Salvia, Solanum (con 7 cada uno), Smilax (5) y Cestrum (4), entre otros.

Cuadro 1. Participación de los diferentes grupos de plantas vasculares en Eloxochitlán y Tlahuelompa, Hidalgo.

\begin{tabular}{|l|r|r|r|}
\hline Taxón & Familias & Géneros & Especies \\
\hline Pteridophyta & $12(10.9 \%)$ & $23(8.6 \%)$ & $31(7.8 \%)$ \\
Gymnospermae & $5(4.5 \%)$ & $5(1.9 \%)$ & $6(1.5 \%)$ \\
Dicotyledonae & $82(74.5 \%)$ & $203(76.3 \%)$ & $311(79 \%)$ \\
Monocotyledonae & $11(10.1 \%)$ & $35(13.2 \%)$ & $46(11.6 \%)$ \\
Total & 110 & 266 & 394 \\
\hline
\end{tabular}

Para Eloxochitlán se obtuvo un listado compuesto por 287 especies, 199 géneros y 93 familias y en Tlahuelompa se recolectaron 233 especies, pertenecientes a 157 géneros y 85 familias. Ambas áreas comparten 126 especies.

Las familias mejor representadas en cada zona en términos del número de especies se enlistan en el Cuadro 2 y sus porcentajes se muestran en la Fig. 4.

Cuadro 2. Familias más abundantes en cuanto a número de especies para cada localidad.

\begin{tabular}{|l|r|l|r|}
\hline Eloxochitlán & & Tlahuelompa & \\
\hline Compositae & 30 & Compositae & 24 \\
Solanaceae & 15 & Solanaceae & 13 \\
Labiatae & 12 & Fagaceae & 10 \\
Leguminosae & 10 & Rubiaceae & 10 \\
Orchidaceae & 10 & Labiatae & 8 \\
Rubiaceae & 9 & Orchidaceae & 8 \\
Fagaceae & 7 & Rosaceae & 8 \\
Rosaceae & 7 & Ericaceae & 6 \\
Aspleniaceae & 6 & & \\
Begoniaceae & 6 & & \\
Urticaceae & 6 & & \\
\hline
\end{tabular}

Los géneros con un mayor número de especies en cada localidad se enlistan en el Cuadro 3; de ellos 90 se comparten entre ambas localidades.

A nivel de especie, las hierbas son más abundantes (176, 44.5\%), seguidas por los árboles $(91,23 \%)$, los arbustos $(69,17.5 \%)$, los bejucos $(33,8.4 \%)$, las epífitas $(23,5.8 \%)$ y las parásitas $(3,0.8 \%)$. El número de especies por forma de vida en cada localidad y el que comparten ambas localidades se muestran en la Fig. 5. 
Alcántara y Luna: Análisis Florístico de dos Áreas con Bosque Mesófilo de Montaña

a
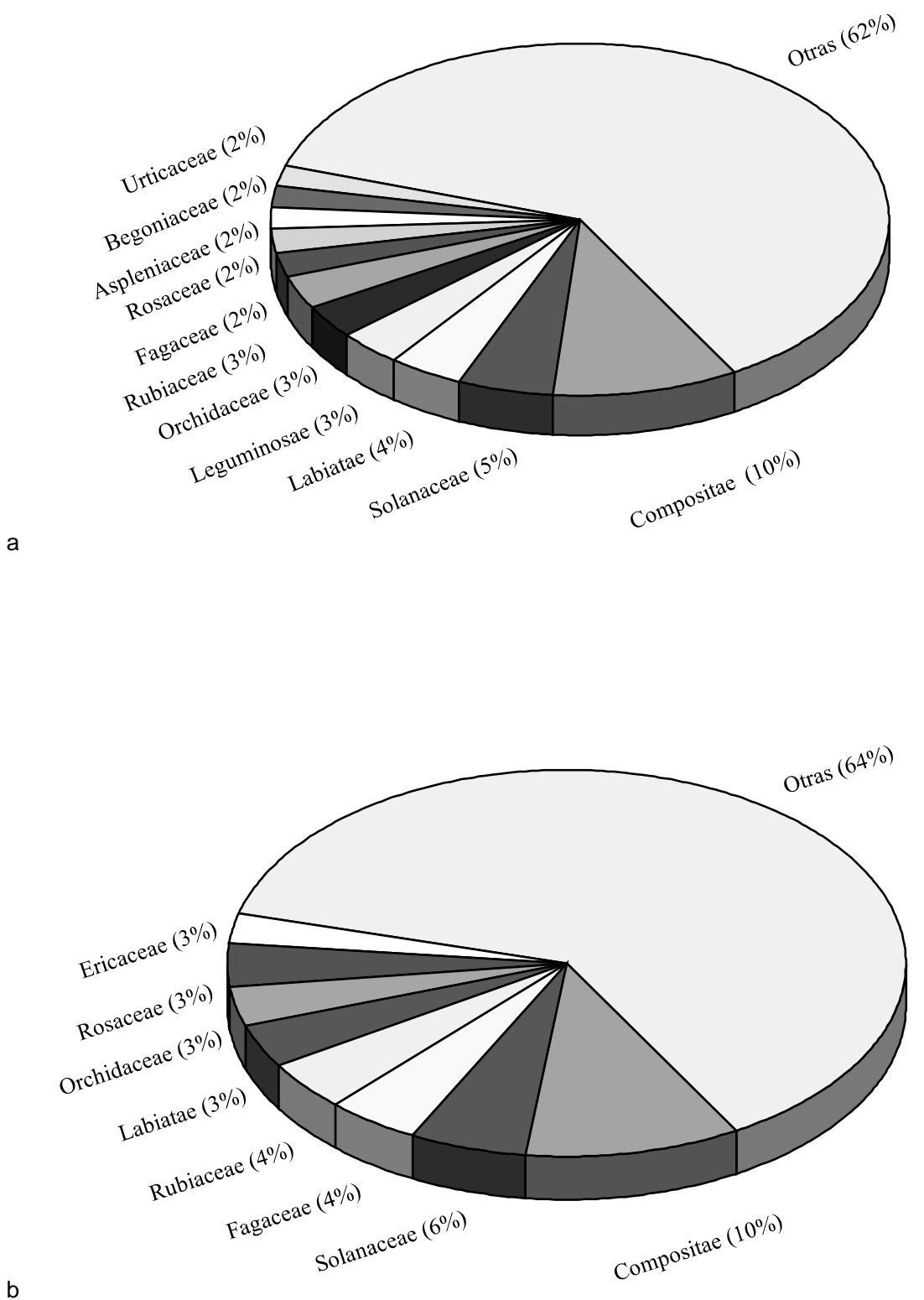

Fig. 4. Familias mejor representadas en cuanto a número de especies en ambas localidades. a) Eloxochitlán; b) Tlahuelompa. 
Cuadro 3. Géneros con mayor número de especies (tres o más) para cada una de las localidades.

\begin{tabular}{|l|r|l|l|}
\hline Eloxochitlán & & Tlahuelompa & \\
\hline Salvia & 10 & Quercus & 9 \\
Quercus & 7 & Salvia & 7 \\
Solanum & 7 & Solanum & 7 \\
Begonia & 6 & Smilax & 5 \\
Ageratina & 4 & Cestrum & 4 \\
Polypodium & 4 & Cuphea & 3 \\
Smilax & 4 & Ipomoea & 3 \\
Dioscorea & 3 & Lobelia & 3 \\
Ipomoea & 3 & Lycopodium & 3 \\
Malaxis & 3 & Piper & 3 \\
Oenothera & 3 & Polypodium & 3 \\
Phenax & 3 & Rhamnus & 3 \\
Prunus & 3 & Viburnum & 3 \\
Rhamnus & 3 & Zanthoxylum & 3 \\
\hline
\end{tabular}

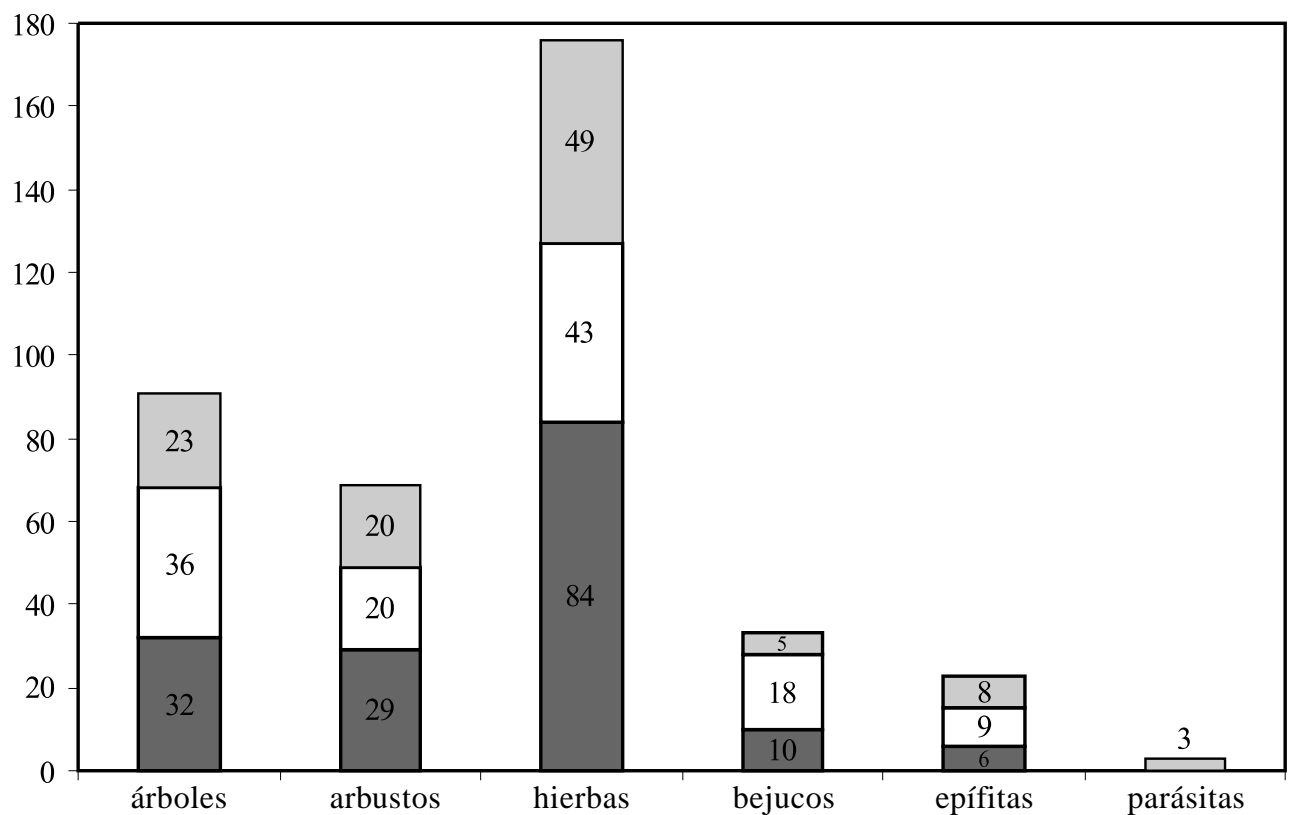

$\square$ Eloxochitlán $\square$ Ambas localidades $\square$ Tlahuelompa

Fig. 5. Abundancia de especies por forma de vida en cada localidad y en ambas localidades. 
Alcántara y Luna: Análisis Florístico de dos Áreas con Bosque Mesófilo de Montaña

Caracterización del bosque

Eloxochitlán

El bosque de la región de Eloxochitlán se presenta principalmente en cañadas en un gradiente altitudinal que va de los 1400 a los 2210 m s.n.m. En casi toda esta extensión se pueden distinguir tres estratos arbóreos. En las partes más bajas y hasta los $1700 \mathrm{~m}$ s.n.m., los árboles altos más importantes (de más de $20 \mathrm{~m}$ ) son Magnolia schiedeana, Meliosma alba, Persea americana, Quercus acutifolia, Quercus sartorii y Tilia houghi; entre los de mediana estatura (de 12 a $20 \mathrm{~m}$ ) destacan Clethra alcoceri, C. mexicana, Ocotea klotzschiana, Styrax glabrescens y entre los bajos (2 a $12 \mathrm{~m}$ ) Ilex tolucana, Perrottetia ovata, Picramnia xalapensis y Saurauia scabrida. Los arbustos más importantes en este intervalo son Ardisia compressa, Eupatorium ligustrinum, Piper spp., Rondeletia capitellata, Senecio arborescens y Solanum spp., entre otros. Las hierbas están representadas por especies de las familias Compositae, Labiatae, Melastomataceae y Rubiaceae principalmente. Los bejucos más importantes son Ipomoea spp. y Smilax spp.

Por arriba de los 1700 m s.n.m. los árboles altos más importantes (más de $20 \mathrm{~m}$ ) son Carpinus caroliniana, Magnolia schiedeana, Quercus affinis, Q. germana, Q. sartorii y Tilia houghi. Entre los de talla mediana (12 a $20 \mathrm{~m})$ sobresalen Bauhinia chapulhuacania, Clethra mexicana y Styrax glabrescens y entre los bajos (menos de $12 \mathrm{~m}$ ) están Crataegus mexicana, Ilex discolor, Picramnia xalapensis, Ptelea trifoliata y Rhamnus pompana. Podocarpus reichei es un árbol de poca estatura y escaso en la zona. En el estrato arbustivo son comunes Cestrum spp., Deppea microphylla, Eupatorium ligustrinum, Piper spp., Randia laetevirens, Roldana aschenborniana y Rondeletia capitellata. A lo largo de casi todo el gradiente, Oreopanax flaccidus es un arbusto epífito importante. El estrato herbáceo está compuesto principalmente de helechos y plántulas de Clethra alcoceri, C. mexicana y Quercus spp. entre otras especies de árboles, aunque en los sitios más abiertos son abundantes diferentes especies de Compositae, Rubiaceae y Solanaceae. Entre los bejucos están Smilax spp., Solandra sp., Vitis popenoei y en zonas más asoleadas Clematis acapulcensis, Dioscorea spp. e Ipomoea spp.

En las laderas con pendiente de orientación norte, aproximadamente a los $1790 \mathrm{~m}$ s.n.m., la composición florística cambia, debido a que estas cuestas están menos protegidas; en el estrato alto (más de $20 \mathrm{~m}$ ) son frecuentes Cupressus benthamii, Pinus patula, $P$. pseudostrobus y Quercus crassifolia y en el arbustivo Deppea microphylla, Eupatorium ligustrinum, Oxalis rhombifolia y Roldana aschenborniana.

Por arriba de los $1900 \mathrm{~m}$ de altitud se observa la alternancia con el bosque de Cupressus, el cual habita en las áreas más expuestas. Más allá de este intervalo (a 2210 $\mathrm{m}$ aproximadamente) prevalece el bosque de Cupressus benthamii propiamente dicho, que se mezcla en algunas zonas con Pinus patula (Fig. 3).

Tlahuelompa

El bosque de Tlahuelompa se presenta como un mosaico de manchones con diferente composición y dominancia; cerca del poblado de Tzincoatlán (1690 m s.n.m.) son conspicuos los siguientes componentes de más de $20 \mathrm{~m}$ de altura: Liquidambar macrophylla, 
Pinus patula, Quercus affinis y $Q$. eugeniifolia, como árboles medianos destacan Clethra alcoceri, C. mexicana y Styrax glabrescens y entre los bajos Cornus disciflora y Zanthoxylum xicense.

En el área cercana al poblado de Tizapán (1960 m s.n.m.) los elementos que dominan el dosel alto (20-30 m de alto) son Liquidambar macrophylla, Quercus affinis, Q. eugeniifolia, $Q$. germana y $Q$. sartorii, en el medio $(12$ a $18 \mathrm{~m}$ ) prevalecen Clethra mexicana y Cleyera theaeoides, y en el bajo Ocotea effusa y Turpinia occidentalis. En el estrato herbáceo son frecuentes Lophosoria quadripinnata, plántulas de Clethra spp. y Melastomataceae.

El área de La Mojonera, de los 1800 a los 2000 m s.n.m., alberga al manchón de bosque de Fagus grandifolia ssp. mexicana más importante del país, en el cual dominan también en el estrato alto (25-40 m) Quercus eugeniifolia, Q. sartorii y $Q$. trinitatis y en algunas zonas expuestas Pinus patula; Liquidambar macrophylla es muy escaso. Entre los árboles medianos se registraron Clethra mexicana, Magnolia schiedeana, Ostrya virginiana y entre los bajos destacan Befaria laevis, Cleyera theaeoides, Ocotea klotzschiana y Turpinia occidentalis. El estrato arbustivo es pobre y su componente más representativo es Eupatorium sp. Los elementos herbáceos son también pocos y están representados por algunas especies de Miconia y de helechos. Las epífitas más conspicuas son de las familias Piperaceae y Orchidaceae, principalmente Rhynchoglossum rossii. El suelo es rico en materia orgánica y hojarasca, principalmente de Fagus. En las laderas más expuestas abundan Befaria laevis y Quercus crassifolia.

Análisis de la distribución geográfica

Los géneros se agruparon en seis categorías de acuerdo con su distribución geográfica actual (Cuadro 4). La mayor proporción restringe su área a América (93, 35\%); los demás son propios de los trópicos y subtrópicos $(56,21.1 \%)$, del hemisferio norte (41, $15.4 \%$ ), de amplia distribución (36, 13.5\%), exclusivos de los trópicos (27, 10.2\%), o bien de zonas templadas y subtropicales (13, 4.8\%).

Siete géneros (Aulosepalum, Ceratozamia, Cobaea, Decatropis, Holodyctium, Smithiantha y Tetrachyron) son endémicos o cuasiendémicos a México (propios de Megaméxico 2 en el sentido de Rzedowski, 1991).

Al agrupar a los géneros por su forma de vida preponderante (Cuadro 4), se encontró que la mayoría de los correspondientes a los árboles se distribuyen principalmente en el hemisferio norte (25), mientras que los propios de las formas biológicas restantes restringen mayormente sus áreas a América (27 géneros de arbustos, 36 de hierbas, 11 de epífitas, 6 de bejucos y 2 de parásitas), principalmente a las zonas tropicales y cálidas del continente (Fig. 6).

\section{DISCUSIÓN Y CONCLUSIONES}

Los bosques de Tlahuelompa y Eloxochitlán del estado de Hidalgo poseen una flora rica y diversa constituida por 394 especies de plantas vasculares, representando $15.8 \%$ de la riqueza florística estimada para este tipo de vegetación en México (Rzedowski, 1991). El conocimiento florístico de los manchones de bosque mesófilo estudiados de ambas zonas 
era hasta este momento sumamente pobre; para Eloxochitlán no existía listado florístico alguno y en Tlahuelompa sólo se habían registrado 52 especies (Puig, 1976). Los bosques de estas zonas pueden considerarse como característicos de este tipo de vegetación en México.

Desde un punto de vista ecológico y considerando aspectos fisonómico-estructurales y florísticos, se reconocen varias asociaciones vegetales determinadas básicamente por el clima (diferencias en el grado de humedad y temperatura) y la orografía (relieve y exposición de la ladera). No obstante, una caracterización del bosque mesófilo de montaña debe tomar en cuenta su complejidad histórica y geográfica, además de la ecológica.

Cuadro 4. Distribución actual de los géneros por forma de vida.

\begin{tabular}{|c|c|c|c|c|c|}
\hline $\begin{array}{l}\text { Templado y } \\
\text { subtropical }\end{array}$ & $\begin{array}{l}\text { Hemisferio } \\
\text { Norte }\end{array}$ & Tropical & $\begin{array}{l}\text { Tropical y } \\
\text { subtropical }\end{array}$ & América & $\begin{array}{l}\text { Amplia } \\
\text { distribución }\end{array}$ \\
\hline $\begin{array}{l}\text { ÁRBOLES } \\
\text { Acer } \\
\text { Euonymus } \\
\text { Sambucus }\end{array}$ & \begin{tabular}{|l} 
Alnus ${ }^{1}$ \\
Arbutus \\
Carpinus \\
Carya \\
Cercis \\
Cercocarpus \\
Cleyera \\
Cornus \\
Crataegus \\
Cupressus \\
Fagus \\
Illicium \\
Juglans \\
Liquidambar \\
Lyonia \\
Magnolia \\
Nyssa \\
Ostrya \\
Pinus \\
Prunus \\
Quercus \\
Rhamnus \\
Taxus \\
Tilia \\
Viburnum
\end{tabular} & $\begin{array}{l}\text { Bauhinia } \\
\text { Lonchocarpus } \\
\text { Meliosma } \\
\text { Microtropis } \\
\text { Persea } \\
\text { Saurauia } \\
\text { Sloanea } \\
\text { Ternstroemia } \\
\text { Trichilia } \\
\text { Trophis }\end{array}$ & $\begin{array}{l}\text { Buddleia } \\
\text { Clethra } \\
\text { Cyathea } \\
\text { Ocotea } \\
\text { Perrottetia } \\
\text { Podocarpus } \\
\text { Styrax } \\
\text { Symplocos } \\
\text { Turpinia }\end{array}$ & $\begin{array}{l}\text { Befaria } \\
\text { Bernardia } \\
\text { Citharexylum } \\
\text { **Decatropis } \\
\text { Dipholis } \\
\text { Heliocarpus } \\
\text { *Lozanella } \\
\text { *Oreopanax } \\
\text { *Picramnia } \\
\text { Ptelea } \\
\text { *Rondeletia }\end{array}$ & $\begin{array}{l}\text { Ilex } \\
\text { Vaccinium } \\
\text { Zanthoxylum }\end{array}$ \\
\hline ARBUSTOS & $\begin{array}{l}\text { Ceanothus } \\
\text { Eupatorium }\end{array}$ & $\begin{array}{l}\text { Lantana } \\
\text { Lippia } \\
\text { Litsea } \\
\text { Miconia } \\
\text { Piper }\end{array}$ & $\begin{array}{l}\text { Ardisia } \\
\text { Fuchsia } \\
\text { Gaultheria } \\
\text { Packera } \\
\text { Vernonia }\end{array}$ & $\begin{array}{l}\text { Ageratina } \\
\text { Archibaccharis } \\
\text { Baccharis } \\
\text { Barkleyanthus } \\
\text { Bocconia }\end{array}$ & $\begin{array}{l}\text { Berberis } \\
\text { Rubus } \\
\text { Senecio } \\
\text { Senna } \\
\text { Solanum }\end{array}$ \\
\hline
\end{tabular}


Cuadro 4. Continuación.

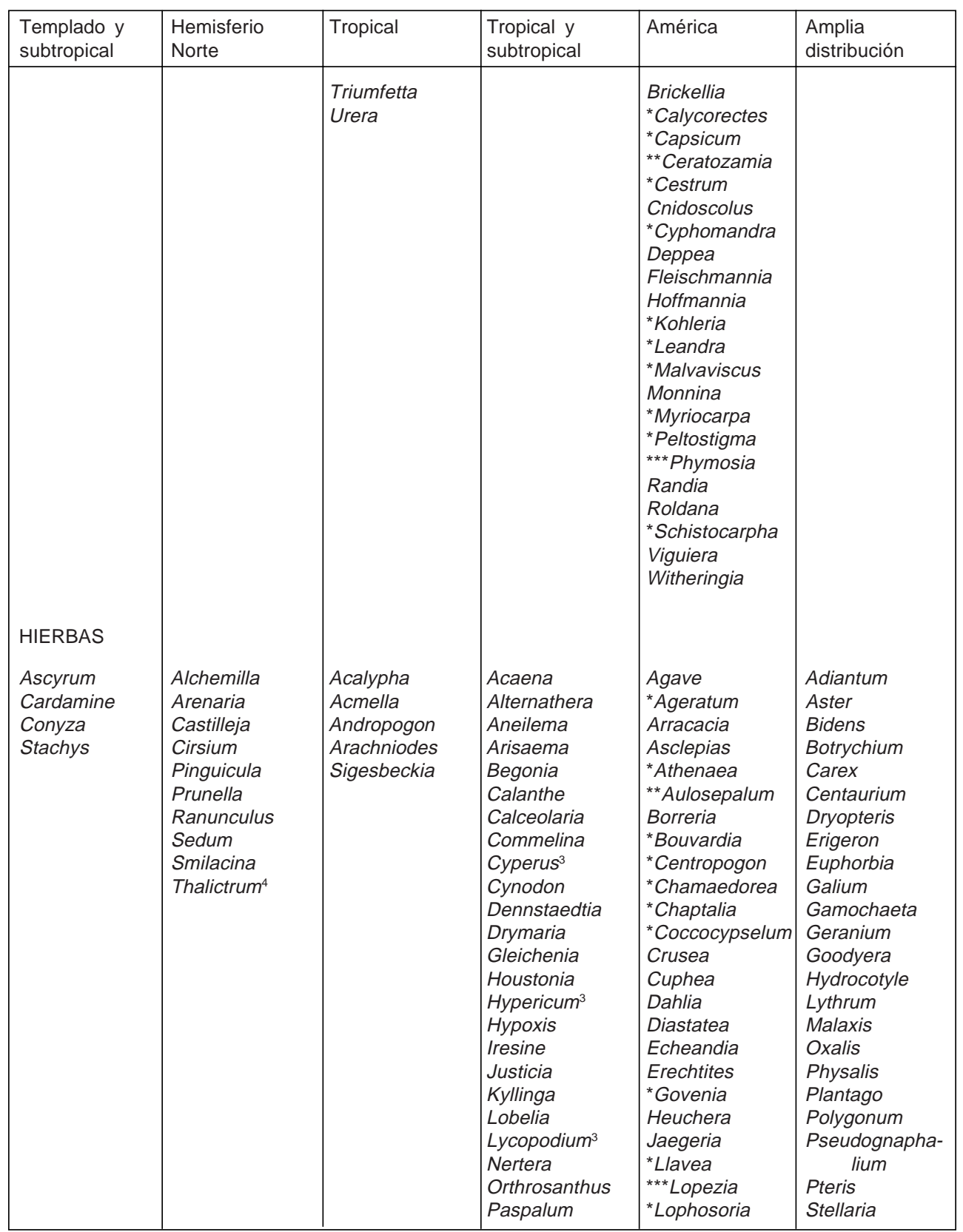


Alcántara y Luna: Análisis Florístico de dos Áreas con Bosque Mesófilo de Montaña

Cuadro 4. Continuación.

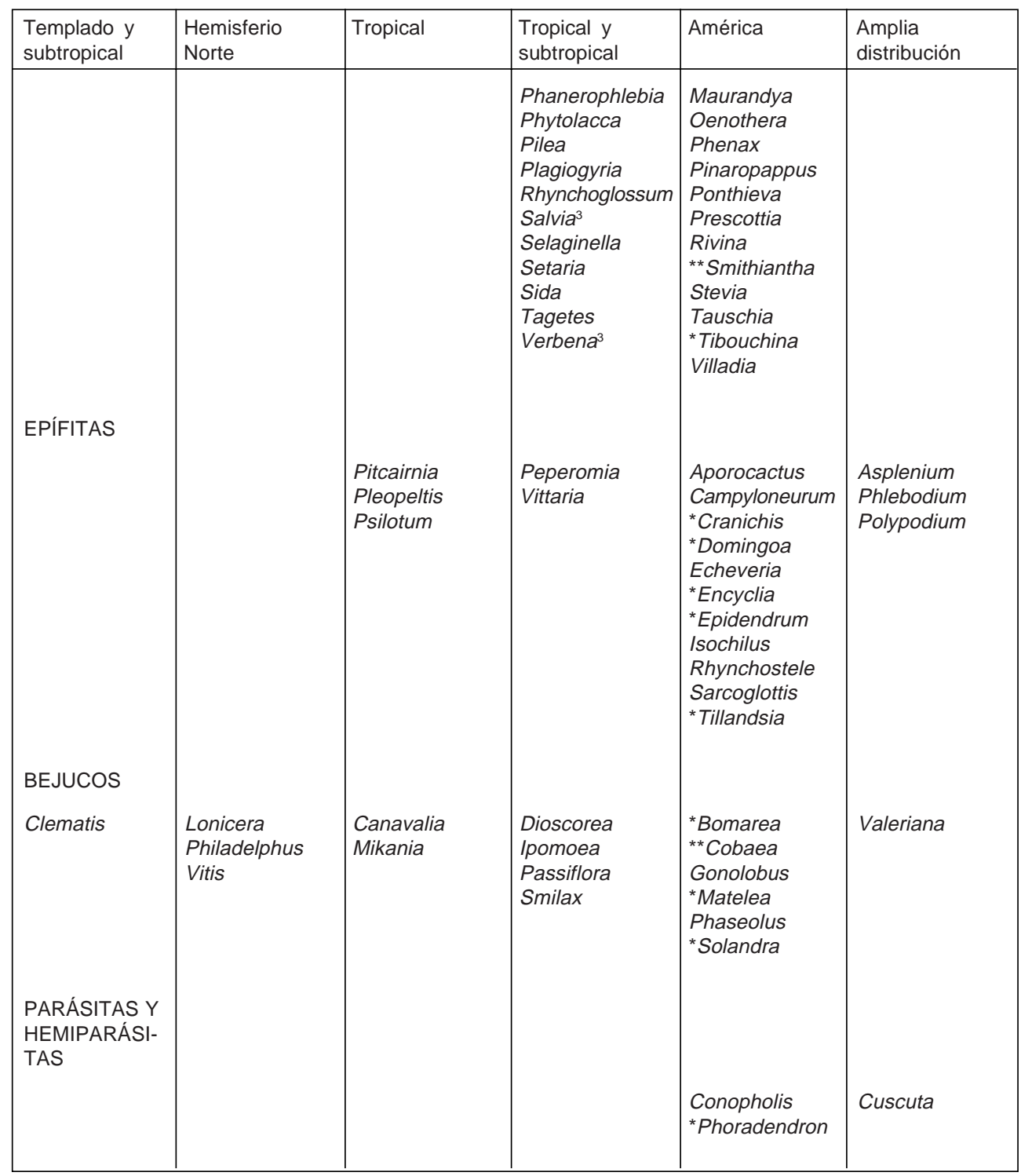

1. con una especie en los Andes, 2. también en los trópicos de Asia y África, 3. también en zonas templadas y frías, 4. también Nueva Guinea, trópicos de América y África, * distribución tropical, ${ }^{* *}$ endémico 0 cuasiendémico de México $y^{* \star *}$ de México a Centroamérica. 


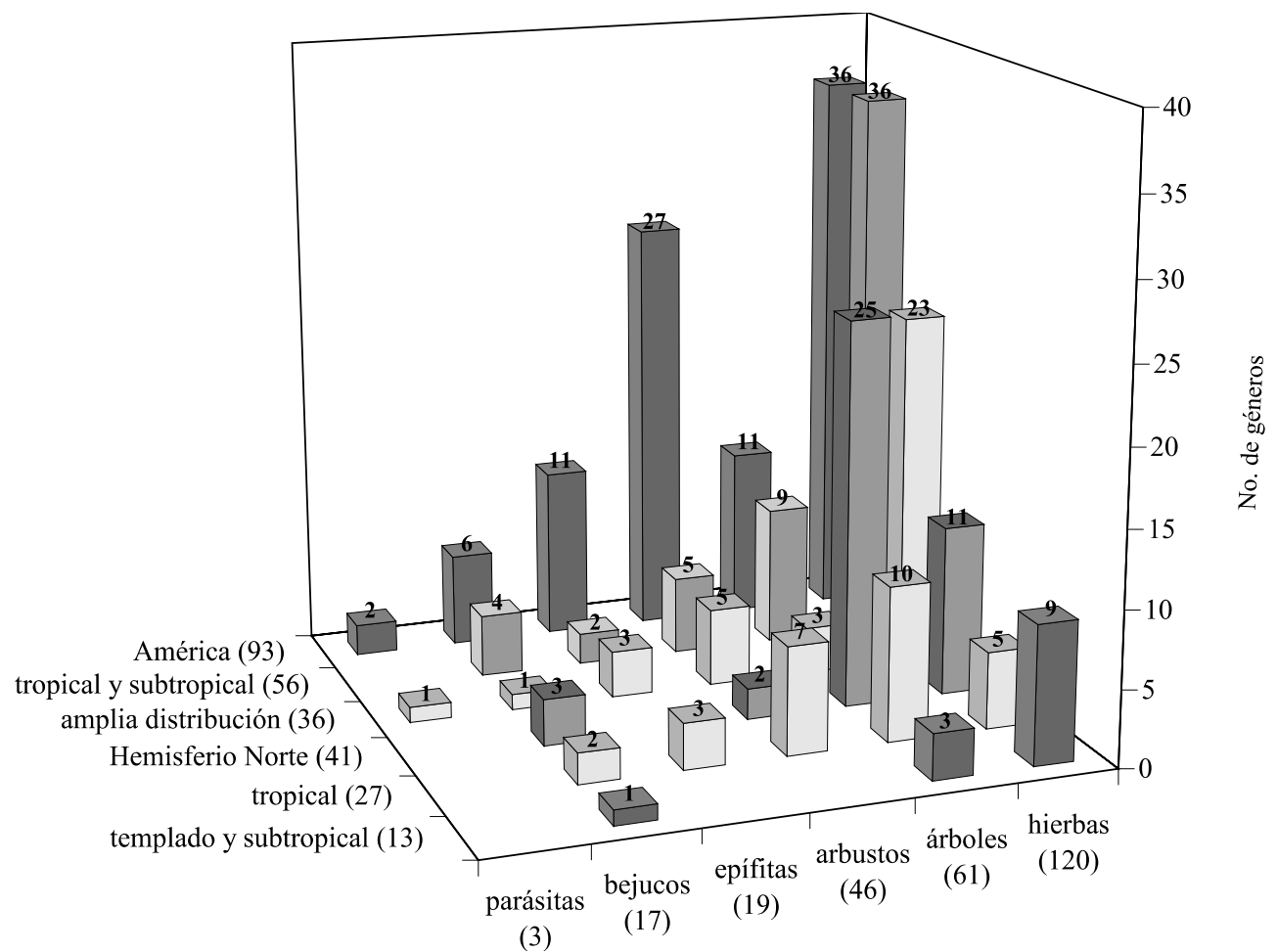

Fig. 6. Distribución actual por forma biológica de los géneros de plantas vasculares de los bosques de Eloxochitlán y Tlahuelompa.

De las familias que Rzedowski (1996) considera que prosperan preferentemente en esta comunidad, en Tlahuelompa y Eloxochitlán se presentan las siguientes: Aceraceae, Aquifoliaceae, Begoniaceae, Clethraceae, Cornaceae, Cyatheaceae, Gesneriaceae, Gleicheniaceae, Lauraceae, Lycopodiaceae, Magnoliaceae, Myrsinaceae, Orchidaceae, Piperaceae, Selaginellaceae, Staphyleaceae, Styracaceae, Symplocaceae, Taxaceae y Theaceae, esto es, $60.6 \%$.

Algunos géneros de árboles cuantitativamente importantes en los bosques de Tlahuelompa y Eloxochitlán son: Alnus, Carpinus, Carya, Clethra, Cleyera, Cornus, Juglans, Liquidambar, Meliosma, Oreopanax, Persea, Prunus, Quercus, Styrax y Ternstroemia, mismos que poseen especies características de este tipo de vegetación.

Algunas especies de distribución restringida y/o en peligro de extinción que se presentan en el área son: Carya palmeri, Ceratozamia mexicana, Fagus grandifolia ssp. 
mexicana, Litsea glaucescens, Magnolia schiedeana, Taxus globosa y Tibouchina galeottiana. Otras consideradas como raras, amenazadas o sujetas a protección especial por la Norma Oficial Mexicana (Anónimo, 2000) son Bouvardia xylosteoides, Carpinus caroliniana y Cyathea fulva.

Los géneros de árboles bajos, arbustos y hierbas tienen una distribución actual principalmente en los trópicos y subtrópicos de América; los de los árboles altos se distribuyen sobre todo en las zonas frías y templadas del hemisferio Norte, con algunas excepciones.

Es evidente, como se ha planteado en diversos trabajos como el de Rzedowski (1996) entre otros y en estudios de biogeografía histórica contemporánea (Luna et al., 1999, Luna et al., 2000), que las superficies cubiertas por el bosque mesófilo de esta región de la Huasteca Hidalguense (Eloxochitlán, Tlahuelompa, Molocotlán, Tlanchinol y Tenango de Doria) conforman fracciones de un manchón más extenso que en el transcurso del tiempo se ha ido fragmentando. Estudios comparativos entre los diversos rodales de este tipo de vegetación en la región (Luna et al. 2000, Luna et al. en prensa), muestran que ambas zonas tienen una composición florística similar a la de los bosques cercanos geográficamente, como los de Tlanchinol, Tenango de Doria y Molocotlán, aunque en ocasiones los elementos dominantes son diferentes, incluso dentro de un mismo manchón, dependiendo de muchos factores como exposición de la ladera, humedad y microclima, entre otros. Por ejemplo, en Tlahuelompa el elemento arbóreo que fisonómicamente domina es Fagus grandifolia ssp. mexicana, pero en ciertos sitios más expuestos son comunes Liquidambar macrophylla y varias especies de encinos.

Al igual que muchos géneros de plantas de los bosques templados del este de México, otros taxones de animales y hongos muestran patrones de distribución disyuntos coincidentes entre el este de Norteamérica y el este de México-Centroamérica (Martin, 1958; Rosen, 1978, Contreras-Medina y Eliosa-León (en prensa), entre otros); esta disyunción permite suponer una continuidad biótica más antigua entre estas áreas y la explicación de los patrones actuales de distribución pueden hacerse con base en ciertos eventos históricogeológicos o geográficos (Rosen, 1978). Algunos ejemplos de plantas con distribuciones disyuntas entre el este de Norteamérica y el este de México, presentes en las zonas de estudio, son Fagus grandifolia, Illicium floridanum, Nyssa sylvatica y Schizandra glabra, esta última registrada sólo para una localidad cercana al bosque Tlahuelompa (Panero y Dávila, 1998). Otro ejemplo notable de distribución disyunta a nivel de especie lo constituye el caso de Meliosma alba, sólo presente en el este de México y Guatemala y el sureste de Asia (Beusekom, 1971; Durán-Espinosa, 1997).

Se pueden citar también varios casos de vicarianza entre las diferentes islas del bosque mesófilo mexicano, entre las que destacan especies de Clethra, Deppea, Magnolia, Meliosma, Randia, Styrax y Symplocos, entre otros (Alcántara et al., en prensa). Este proceso probablemente se ha debido a que los fenómenos orogénicos, tanto tectónicos como volcánicos, junto con las fluctuaciones climáticas pasadas, han jugado un papel importante en los procesos de vicarianza, generando a su vez la fragmentación de poblaciones ancestrales y produciendo eventos de especiación en varios taxa (Ramamoorthy y Lorence, 1987).

Los bosques de Tlahuelompa y Eloxochitlán, al igual que otros bosques montanos, se encuentran sometidos a fuertes presiones humanas v. gr. extracción de madera, 
agricultura y ganadería. La tala de árboles altos de manera clandestina se lleva a cabo para la obtención de leña. Esto puede dar como consecuencia el elevado número de especies de las familias Compositae y Solanaceae, así como la relativa escasez de otras como Orchidaceae, Bromeliaceae y Selaginellaceae, que suelen estar mucho mejor representadas en este tipo de vegetación. Algunos taxones que, aunque pueden ser parte del bosque pristino, llegan a ser abundantes en condiciones de aclareamiento son: Alnus jorullensis, Cornus disciflora, Crataegus mexicana, Decatropis bicolor, Lophosoria quadripinnata, Ptelea trifoliata, Prunus serotina, Rubus adenotrichus, Saurauia scabrida, Solanum aligerum y Trema micrantha, entre otros.

Conservación del bosque de Fagus

De acuerdo con el registro fósil, Fagus grandifolia existió en el este de Asia durante el Oligoceno Tardío y en el oeste de Norteamérica (incluyendo Alaska) durante el OligocenoMioceno Tardío (Pérez, 1994), pero en la actualidad su distribución se restringe al este de Norteamérica (sureste de Canadá y Estados Unidos) y pequeños parches de los estados de Tamaulipas, Hidalgo, Veracruz y Puebla, en México, los cuales sin duda representan áreas relictuales dignas de una mayor atención en estudios biogeográficos y de conservación.

A nivel nacional se considera que Fagus grandifolia var. mexicana está en peligro de extinción, ya que su número es inferior a 20,000 individuos (Pérez, 1999). La cantidad de individuos sigue decreciendo, debido en parte a la explotación clandestina y disturbio antropogénico que se ha venido dando en los últimos años. La densidad de la población también se ve afectada por el ciclo de vida tan largo de la especie; este árbol produce semillas cada siete años y la viabilidad de éstas es delicada. Por los factores anteriores, se considera importante continuar con la veda de este taxon, misma que ha permanecido por 40 años, para lo cual se requiere que se mantenga y cumpla la legislación del uso de la tierra.

Pérez (1994) llevó a cabo estudios fitosanitarios de este taxon y con base en ellos considera que la población más grande y con mayor heterogeneidad genética se encuentra en Hidalgo (Montes de Zacatlamaya), por lo que se sugiere proponer un área de reserva (conservación in situ) en este rodal, mismo que actualmente sólo contiene aproximadamente 10,000 individuos.

\section{AGRADECIMIENTOS}

Se agradece a las siguientes personas su ayuda en la determinación del material: Ramiro Cruz y Gabriel Flores (Leguminosae), Abisaí García (Amaryllidaceae), Martha Martínez (Euphorbiaceae), José Luis Villaseñor y Miguel Luna (Compositae), Alejandro Miranda (Gramineae), Nelly Diego (Cyperaceae), Susana Valencia (Fagaceae), Gerardo Salazar (Orchidaceae) y Mónica Palacios Ríos (Pteridophyta y grupos afines). Raúl Contreras y David Espinosa hicieron valiosas observaciones al manuscrito; asimismo dos árbitros anónimos y los editores de la revista nos hicieron excelentes sugerencias que enriquecieron nuestro trabajo. 


\section{LITERATURA CITADA}

Alcántara, O. e I. Luna. 1997. Florística y análisis biogeográfico del bosque mesófilo de montaña de Tenango de Doria, Hidalgo, México. Anales Inst. Biol. Univ. Nac. Autón. México, Ser. Bot. 68(2): 57-106.

Alcántara, O., I. Luna y A. Velázquez. (en prensa). Altitudinal distribution patterns of Mexican cloud forest based upon preferential characteristic genera. Plant Ecology.

Anónimo. 1983. Carta geológica 1: 250,000. Pachuca F1411. Secretaría de Programación y Presupuesto. México, D.F.

Anónimo. 1989. Carta topográfica 1:50,000. Molango F14D51. Secretaría de Programación y Presupuesto. México, D.F.

Anónimo. 1991. Carta topográfica. 1:50,000. Zacualtipán F14D62. Instituto Nacional de Estadística, Geografía e Informática. México, D.F.

Anónimo. 1992a. Síntesis geográfica del estado de Hidalgo. Instituto Nacional de Estadística, Geografía e Informática. México, D.F. 134 pp.

Anónimo. 1992b. Monografía geológico-minera del estado de Hidalgo. Consejo de Recursos Minerales. Subsecretaría de Energía, Minas e Industria Paraestatal. Subsecretaría de Minas e Industria Básica. México, D.F. 95 pp.

Anónimo. 1996. Anuario estadístico del estado de Hidalgo. Instituto Nacional de Estadística Geografía e Informática. Aguascalientes. 532 pp.

Anónimo. 1997. Carta de climas México. Sistema de Köppen modificado por E. García. Escala 1:1,000,000. Comisión Nacional para el Conocimiento y Uso de la Biodiversidad-Estadigrafía. México, D.F.

Anónimo. 2000. Proyecto de Norma Oficial Mexicana PROY-NOM-059-ECOL-2000, protección ambiental-especies de flora y fauna silvestres de México-Categorías de riesgo y especificaciones para su inclusión, exclusión o cambio. Lista de especies en riesgo. Diario Oficial de la Federación. 16 de octubre del 2000.

Beusekom, Van C.F. 1971. Revisión of Meliosma (Sabiaceae), section Lorenzanea excepted, living and fossil, geography and phylogeny. Blumea 19(3): 355-529.

Brummitt, R. K. y C. E. Powell (eds.). 1992. Authors of plant names. Royal Botanic Gardens, Kew. 732 pp.

Contreras-Medina, R. y H. Eliosa-León. (en prensa). Una visión panbiogeográfica de México. In: Llorente, J. y J. J. Morrone (eds.). Biogeografía de América Latina. Fondo de Cultura Económica. México, D.F.

Durán-Espinosa, C. 1997. Sabiaceae. In: Sosa, V. (ed.). Flora de Veracruz. Fascículo 96. Instituto de Ecología A. C. y University of California, Riverside. Xalapa, Veracruz. 15 pp.

Ehnis, D. A. 1981. Fagus mexicana Martínez, su ecología e importancia. Tesis de licenciatura. Facultad de Ciencias, Universidad Nacional Autónoma de México. México, D.F. 123 pp.

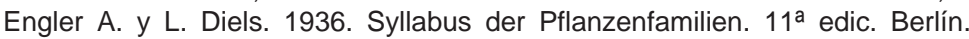

García, E. 1988. Modificaciones al sistema de clasificación climática de Köppen (para adaptarlo a las condiciones de la República Mexicana). Cuarta edición. Universidad Nacional Autónoma de México. México, D.F. 217 pp.

Humphries, C. J., R. I. Vane-Wright y P. H. Williams. 1991. Biodiversity reserves: Setting new priorities for the conservation of wildlife. Parks 2: 34-38.

Liebherr, J. K. 1991. A general area cladogram for montane Mexico based on distributions in the Platynine genera Elliptoleus and Calanthus (Coleoptera: Carabidae). Proc. Ent. Soc. Washington 93: $390-406$.

Luna, I., S. Ocegueda y O. Alcántara. 1994. Florística y notas biogeográficas del bosque mesófilo de montaña del municipio de Tlanchinol, Hidalgo, México. Anales Inst. Biol. Univ. Nac. Autón. México, Ser. Bot. 65(1): 31-62. 
Luna, I., O. Alcántara, D. Espinosa y J. J. Morrone. 1999. Historical relationships of the Mexican cloud forest: A preliminary vicariance model applying parsimony analysis of endemicity to vascular plant taxa. J. Biogeogr. 26(6): 1299-1306.

Luna, I., O. Alcántara, J. J. Morrone y D. Espinosa. 2000. Track analysis and conservation priorities in cloud forest from Hidalgo (Mexico). Diversity and Distributions 6: 137-143.

Luna, I., O. Alcántara y J. J. Morrone. Floristic diversity and generic distributional patterns of the cloud forest of Hidalgo, Mexico. Biotropica (en prensa).

Mabberley, D. J. 1997. The plant-book. A portable dictionary of the vascular plants. Cambridge University Press. Cambridge. $858 \mathrm{pp}$.

Martin, P. S. 1958. A biogeography of reptiles and amphibians in the Gomez Farias region, Tamaulipas, Mexico. Misc. Publ. Mus. Zool. Univ. Michigan 101: 1-102.

Matuda, E. 1953. Plantas asiáticas en México. Mem. Congr. Cient. Mex. 6: 230-248.

Mayorga, R., I. Luna y O. Alcántara. 1998. Florística del bosque mesófilo de montaña de Molocotlán, Molango-Xochicoatlán, Hidalgo, México. Bol. Soc. Bot. México 63: 101-119.

Mickel J. y J. M. Beitel. 1988. Pteridophyte flora of Oaxaca, México. Mem. New York Bot. Gard. 46: 1-566.

Miranda, F. y A. J. Sharp. 1950. Characteristics of the vegetation in certain temperate regions of eastern Mexico. Ecology 31(3): 313-333.

Morrone, J. J., D. Espinosa, C. Aguilar y J. Llorente. 1999. Preliminary classification of the Mexican biogeographic provinces: A parsimony analysis of endemicity based on plant, insect, and bird taxa. The Southwestern Natur. 44 (4): 507-514.

Ochoa-Camarillo, H. R. 1997. Geología del anticlinorio de Huayacocotla en la región de Molango, Hgo., México. Instituto de Investigaciones en Ciencias de la Tierra de la Universidad Autónoma del Estado de Hidalgo e Instituto de Geología de la Universidad Nacional Autónoma de México, II Convención sobre la Evolución Geológica de México y Recursos Asociados, Pachuca, Hgo., Libro-guía de las excursiones geológicas, Excursión 1, pp. 1-17.

Ortega, F. y G. Castillo. 1996. El bosque mesófilo de montaña y su importancia forestal. Ciencias 43: 32-39.

Panero, J. L. y P. Dávila. 1998. The family Schisandraceae: A new record for the flora of Mexico. Brittonia 50(1): 87-90.

Pérez, P. M. 1994. Revisión sobre el conocimiento dendrológico, silvícola y un censo de las poblaciones actuales del género Fagus en México. Tesis de Maestría (Biología). Facultad de Ciencias. Universidad Nacional Autónoma de México. México, D.F. 146 pp.

Pérez, P. M. 1999. Las hayas de México. Monografía de Fagus grandifolia spp. mexicana. Universidad Autónoma Chapingo. Chapingo, México. 51 pp.

Puig, H. 1976. Végétation de la Huasteca, Mexique. Mission Archéologique et Ethnologique Française au Mexique. México, D.F. 531 pp.

Puig, H. 1989. Análisis fitogeográfico del bosque mesófilo de montaña de Gómez Farías, Tamaulipas, México. Biotam 1(2): 34-53.

Ramamoorthy T. P. y D. H. Lorence. 1987. Species vicariance in the Mexican flora and description of a new species of Salvia (Lamiaceae). Bull. Mus. Nat. Hist. Nat., París, $4^{e}$ sér., 9, section B, Adansonia No 2: 167-175.

Rosen, D. E. 1978. Vicariant patterns and historical explanation in biogeography. Syst. Zool. 27: 159188.

Rzedowski, J. 1978. Vegetación de México. Editorial Limusa. México, D.F. 432 pp.

Rzedowski, J. 1991. Diversidad y orígenes de la flora fanerogámica de México. Acta Bot. Mex. 14: 321.

Rzedowski, J. 1996. Análisis preliminar de la flora vascular de los bosques mesófilos de México. Acta Bot. Mex. 35: 25-44.

Sharp, A. J. 1953. Notes on the flora of Mexico: world distribution of the woody dicotyledoneous families and the origin of the modern vegetation. J. Ecol. 41: 374-380. 
Sharp, A. J. 1966. Some aspects of Mexican phytogeography. Ciencia (México) 24: 229-232.

Sharp, A. J. y Z. Iwatsuki. 1965. A preliminary statement concerning mosses common to Japan and Mexico. Ann. Missouri Bot. Gard. 52 (3): 452-456.

Williams, P. H. y C. J. Humphries. 1994. Cap. 19. Biodiversity, taxonomic relatedness, and endemism in conservation. In: Forey, P. I., C. J. Humphries y R. I. Vane-Wright (eds.). Systematics and Conservation Evaluation. Systematics Association Special Volume No. 50, Clarendon Press. Oxford. pp. 269-287.

Willis, J. C. 1973. A dictionary of the flowering plants and ferns. Eighth edition. Cambridge University Press. Cambridge. 1245 pp. 


\section{APÉNDICE}

Lista florística del bosque mesófilo de montaña de Eloxochitlán y Tlahuelompa, Hgo. Aa=árbol

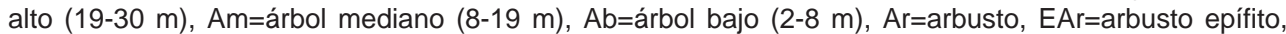
$\mathrm{H}=$ hierba, Be=bejuco, Ep=epífita y $\mathrm{P}=$ parásita o hemiparásita; Eloxochitlán (1) y Tlahuelompa (2).

\section{PTERIDOPHYTA}

\section{ADIANTACEAE}

H. Adiantum andicola Liebm. 1, 2

H. Llavea cordifolia Lag. 1

H. Pteris orizabae M. Martens et Galeotti 1

Ep. Vittaria graminifolia Kaulf. 2

\section{ASPLENIACEAE}

H. Arachniodes denticulata (Sw.) Ching 1

$\mathrm{H} . \quad$ Asplenium cuspidatum Lam. 1

H. A. sessilifolium Desv. 1, 2

H. Dryopteris wallichiana (Spreng.) Hyl. 1, 2

Ep. Holodictyum ghiesbreghtii (Fourn.) Maxon 2

H. Phanerophlebia macrosora (Baker) Underw. 1

H. P. remotispora Fourn. 1

\section{CYATHEACEAE}

Ab. Cyathea fulva (M. Martens et Galeotti) Fée 1, 2

Ab. C. mexicana Schltdl. et Cham. 1

\section{DENNSTAEDTIACEAE}

$\mathrm{H}$. Dennstaedtia dissecta (Sw.) Moore 1

Ar. Saccoloma inaequale (Kunze) Mettenius 1

\section{GLEICHENIACEAE}

H. Gleichenia brevipubis C. Chr. 2

\section{LOPHOSORIACEAE}

Ar. Lophosoria quadripinnata (Gmelin) C. Chr. 2

\section{LYCOPODIACEAE}

H. Lycopodium cernuum L. 2

H. L. clavatum L. 2

H. L. thyoides Humb. et Bonpl. 2

OPHIOGLOSSACEAE

H. Botrychium virginianum (L.) Sw. 1

\section{PLAGIOGYRIACEAE}

H. Plagiogyria semicordata (Presl) Ching 2

\section{POLYPODIACEAE}

Ep. Campyloneurum angustifolium (Sw.) Fée 2 
Apéndice. Continuación.

Ep. Phlebodium areolatum (Humb. et Bonpl.) J. Sm. 2

Ep. Pleopeltis interjecta (Weath.) Mickel et Beitel 1, 2

Ep. Polypodium longepinnulatum Fournier 1, 2

H. $\quad$ P. plebeium Schltdl. et Cham. 1, 2

Ep. $\quad$ P. rhodopleuron Kunze 1

H. Polypodium sp. 1, 2

PSILOTACEAE

Ep. Psilotum complanatum Sw. 2

SELAGINELLACEAE

H. Selaginella sp. 2

\section{GYMNOSPERMAE}

CUPRESSACEAE

Aa. Cupressus benthamii Endl. 1

CYCADACEAE

Ar. Ceratozamia mexicana Brongn. 1

PODOCARPACEAE

Am. Podocarpus reichei Buchholz et Gray 1

PINACEAE

Aa. Pinus patula Schiede et Deppe ex Schltdl. et Cham. 1, 2

Aa. Pinus pseudostrobus Lindl. 1

TAXACEAE

Ab. Taxus globosa Schltdl. 1

\section{ANGIOSPERMAE}

\section{ACANTHACEAE}

H. Justicia macrantha Benth. 1

$\mathrm{H} . \quad$ Justicia $\mathrm{sp} .1$

ACERACEAE

Am. Acer negundo L. ssp. mexicana (DC.) Wesm. 1, 2

AGAVACEAE

H. Agave celsii Hook. 1

AMARANTHACEAE

Be. Alternanthera microcephala (Moq.) Schinz 1, 2

H. Iresine celosia L. 1, 2 
Apéndice. Continuación.

Be. I. interrupta Benth. 1

AMARYLLIDACEAE

Be. Bomarea acutifolia (Link et Otto) Herb. 1, 2

H. Hypoxis mexicana Schult. f. 1, 2

AQUIFOLIACEAE

Ab. Ilex discolor Hemsl. 1, 2

Ab. I. tolucana Hemsl. 1

ARACEAE

H. Arisaema macrospathum Benth. 1, 2

ARALIACEAE

EAr. Oreopanax flaccidus Marchal 1, 2

Ab. O. xalapensis (Kunth) Decne. et Planch. 1, 2

\section{ASCLEPIADACEAE}

H. Asclepias pellucida Fourn. 1

Be. Gonolobus macranthus Kunze 1

Be. Matelea velutina (Schltdl.) Woodson 1

\section{BALSAMINACEAE}

H. Impatiens sultonii Hook. f. 2

\section{BEGONIACEAE}

$\mathrm{H}$. Begonia fischeri Schrank 1

H. B. gracilis Kunth 1

H. $\quad$ B. maculata Raddi 1

H. B. pinetorum A. DC. 1,2

H. B. plebeja Liebm. 1

H. Begonia sp. 1, 2

\section{BERBERIDACEAE}

Ar. Berberis hartwegii Benth. 1

Ab. B. tenuifolia Lindl. 2

\section{BETULACEAE}

Am. Alnus jorullensis Kunth subsp. lutea Furlow 1, 2

Am. Carpinus caroliniana Walter 1, 2

Aa. Ostrya virginiana (Mill.) K. Koch 1, 2

\section{BROMELIACEAE}

Ep. Pitcairnia ringens Klotzsch 1

Ep. Tillandsia usneoides L. 1, 2

Ep. Tillandsia sp. 1 
Apéndice. Continuación.

CACTACEAE

Ep. Aporocactus flagriformis (Zucc.) Lemaire 1, 2

CAMPANULACEAE

H. Centropogon grandidentatus (Schltdl.) Zahlbr. 2

H. Diastatea tenera (A. Gray) McVaugh 1

H. Lobelia laxiflora Kunth 1,2

H. L. sartorii Vatke 1, 2

H. L. stenodonta (Fern.) McVaugh 2

H. Lobelia sp. 1

CAPRIFOLIACEAE

Be. Lonicera mexicana (Kunth) Rehder 2

Am. Sambucus mexicana C. Presl ex DC. 1, 2

Ab. Viburnum ciliatum Greenm. 2

Ab. V. stellatum L. 2

Ab. V. tiliaefolium (Oerst.) Hemsl. 1, 2

\section{CARYOPHYLLACEAE}

H. Arenaria bourgaei Hemsl. 1

H. A. lycopodioides Willd. ex Schltdl. 1

H. Drymaria villosa Cham. et Schltdl. 2

H. Stellaria ovata Willd. ex Schltdl. 2

H. S. prostrata Ellis 1,2

\section{CELASTRACEAE}

Ab. Euonymus mexicanus Benth. 1

Ab. Microtropis schiedeana Loes. 2

Ab. Perrottetia ovata Hemsl. 1

\section{CLETHRACEAE}

Am. Clethra alcoceri Greenm. 1, 2

Am. C. mexicana A. DC. 1, 2

\section{COMMELINACEAE}

H. Aneilema geniculata (Jacq.) Woodson 1

H. Commelina diffusa Burm. f. 1

H. C. pallida Willd. 1

\section{COMPOSITAE}

H. Acmella oppositifolia (Lam.) R. K. Jansen var. oppositifolia 1, 2

$\mathrm{H}$. Ageratina aschenborniana (Schauer) R. King et H. Rob. 1

Ar. A. pazcuarensis (Kunth) R. King et $\mathrm{H}$. Rob 1

Ar. A. pichinchensis (Kunth) R. King et H. Rob. 1

Ar. A. saltillensis (B. L. Rob.) R. King et H. Rob. 2

Ar. A. subinclusa (Klatt) R. King et $\mathrm{H}$. Rob. 1

$\mathrm{H}$. A. vernalis (Vatke et Kurtz) R. King et $\mathrm{H}$. Rob. 2 
Apéndice. Continuación.

H. $\quad$ Ageratum corymbosum Zuccagni f. euryphyllum (B. L. Rob.) M. F. Johnson 2

Ar. Archibaccharis intermedia (S. F. Blake) B. L. Turner 1, 2

$\mathrm{H}$. Aster moranensis Kunth 2

Ar. Baccharis conferta Kunth 1, 2

Ar. Barkleyanthus salicifolius (Kunth) H. Rob. et Brettell 1

H. Bidens odorata Cav. 1, 2

H. B. schaffneri (A. Gray) Sherff 2

Ar. Brickellia glandulosa (La Llave) McVaugh 1, 2

H. Chaptalia madrensis G. L. Nesom 1

H. $\quad$ Cirsium jorullense (Kunth) Spreng. 1, 2

$\mathrm{H} . \quad$ Conyza canadensis (L.) Cronquist 1

H. Dahlia coccinea Cav. 1

H. Erechtites valerianifolia (Wolf) DC. 2

H. Erigeron karvinskianus DC. 1, 2

Ar. Eupatorium ligustrinum DC. 1, 2

Ar. Fleischmannia pycnocephala (Less.) R. M. King et H. Rob. 2

H. Gamochaeta americana (Mill.) Wedd. 2

H. Jaegeria hirta (Lag.) Less 1, 2

Be. Mikania cordifolia (L.f.) Willd. 1, 2

Ar. Packera sanguisorbae (A. DC.) C. Jeffrey 1, 2

H. Pinaroppapus roseus (Less.) Less. 1

$\mathrm{H}$. Pseudognaphalium attenuatum (DC.) A. Anderb. 1, 2

Ar. Roldana aschenborniana (Schauer) H. Rob. et Brettell 1, 2

Ar. Schistocarpha kellermanii Rydb. 1

Ab. Senecio arborescens Steetz 1

$\mathrm{H}$. Sigesbeckia jorullensis Kunth 1

$\mathrm{H}$. Stevia jorullensis Kunth 2

Ar. S. lucida Lag. 1

H. S. ovata Willd. 1

H. Tagetes erecta L. 2

H. Tetrachyron orizabaensis Sch. Bip. ex Klatt var. websteri (Wussow et Urbatsch) Wussow et Urbatsch 1

Ar. Vernonia deppeana Less. 2

Ar. V. leiboldiana Schltdl. 1

Ar. Viguiera cordata (Hook. et Arn.) D'Arcy var. cordata 1

\section{CONVOLVULACEAE}

P. Cuscuta tinctoria Mart. ex Engelm. 2

Be. Ipomoea indica (Burm. f.) 1, 2

Be. I. purga (Wender.) Hayne 1, 2

Be. I. purpurea (L.) Roth 1, 2

\section{CORNACEAE}

Ab. Cornus disciflora Moc. et Sessé ex DC. 1, 2

Ab. C. excelsa Kunth 1,2

\section{CRASSULACEAE}

Ep. Echeveria aff. guatemalensis Rose 1, 2 
Apéndice. Continuación.

H. E. mucronata (Bak.) Schltdl. 2

H. Sedum greggii Hemsl. 1

H. Villadia sp. 1

CRUCIFERAE

H. Cardamine flaccida Cham. et Schltdl. 1

CYPERACEAE

H. Carex distentiformis F.J. Hermann 1

H. Cyperus hermaphroditus (Jacq.) Standl. 2

$\mathrm{H} . \quad$ C. tenuis Sw. 2

H. Kyllinga pumila Michx. 2

DILLENIACEAE

Ar. Saurauia scabrida Hemsl. 1

DIOSCOREACEAE

Be. Dioscorea convolvulacea Schltdl. et Cham. 1

Be. D. nelsonii Uline ex R. Knuth 1,2

Be. D. mexicana Scheidw. 1, 2

ELAEOCARPACEAE

Ab. Sloanea sp. 1

\section{ERICACEAE}

Am. Arbutus xalapensis Kunth 2

Am. Befaria laevis Benth. 2

Ar. Gaultheria hirtiflora Benth. 2

Ar. G. odorata Bredem. ex Willd. 2

Ab. Lyonia squamulosa M. Martens et Galeotti 2

Am. Vaccinium leucanthum Cham. et Schltdl. 2

\section{EUPHORBIACEAE}

H. Acalypha aff. subviscida Wats. 1

Ar. A. microcephala (Moq.) Schinz 1

Ab. Bernardia mollis Lundell 1

Ar. Cnidoscolus multilobus (Pax) I. M. Johnst. 2

H. Euphorbia sp. 1

\section{FAGACEAE}

Aa. Fagus grandifolia Ehrh. ssp. mexicana (Martínez) E. Murray 2

Am. Quercus acutifolia Née 1

Aa. Q. affinis Scheidw. 1, 2

Ab. Q. crassifolia Humb. et Bonpl. 1, 2

Aa. $Q$. eugeniifolia Liebm. 2

Aa. $Q$. aff. eugeniifolia Liebm. 2

Am. Q. germana Schltdl. et Cham. 1, 2

Am. Q. glabrescens Benth. 2 
Apéndice. Continuación.

Ab. Q. laeta Liebm. 1

Aa. Q. sartorii Liebm. 1, 2

Am. Q. trinitatis Trel. 2

Am. Q. xalapensis Humb. et Bonpl. 1, 2

GENTIANACEAE

H. Centaurium brachycalyx Standl. 1

H. C. chironioides (Griseb.) Druce 2

\section{GERANIACEAE}

H. Erodium sp. 2

H. Geranium seemannii Peyr. 1

\section{GESNERIACEAE}

Ar. Kohleria deppeana (Schltdl. et Cham.) Fritsch 2

H. Rhynchoglossum azureum (Schltdl.) Burtt 1

H. Smithiantha zebrina (Paxton) Kuntze 1, 2

\section{GRAMINEAE}

H. Andropogon aff. glomeratus (Walt.) B. S. P. 1

H. Cynodon dactylon (L.) Pers. 2

H. Paspalum affine Steud. 2

H. Poa anua L. 2

H. Setaria scandens Schrad. 1

\section{GUTTIFERAE}

H. Ascyrum hypericoides L. 2

\section{HAMAMELIDACEAE}

Aa. Liquidambar macrophylla Oerst. 2

\section{ILLICIACEAE}

Ab. Illicium floridanum Ellis 1

\section{IRIDACEAE}

H. Orthrosanthus chimboracensis (Kunth) Baker var. exsertus R. C. Foster 1, 2

\section{JUGLANDACEAE}

Ab. Carya palmeri W. E. Manning 1

Am. Juglans mollis Engelm. 1, 2

\section{LABIATAE}

H. Prunella vulgaris L. 1,2

H. Salvia elegans Vahl 1, 2

$\mathrm{H}$. S. gracilis Benth. 1

H. S. helianthemifolia Benth. 1, 2

H. S. involucrata Cav. 1, 2

H. S. mexicana L. 1 
Apéndice. Continuación.

H. S. microphylla Kunth var. neurepia Epling 1

H. S. polystachya Ortega 1, 2

H. S. simulans Fern. 1, 2

H. S. tiliaefolia Vahl 1,2

H. Salvia sp. 1, 2

H. Stachys sanchezii Rzed. et A. García 1

\section{LAURACEAE}

Ab. Litsea glaucescens Kunth 2

Ab. Ocotea klotzschiana (Nees) Hemsl. 1, 2

Aa. Persea americana Miller 1

Ab. $\quad P$. chamissonis Mez 2

\section{LEGUMINOSAE}

Am. Bauhinia chapulhuacania Wunderlin 1

Ab. B. dipetala Hemsl. 1

Be. Canavalia sp. 1

Am. Cercis canadensis L. 1

Ab. Lonchocarpus sp. 1

H. Melilotus indica (L.) All. 1

Be. Phaseolus coccineus L. 1

Be. $\quad$ P. vulgaris L. 2

Ab. Senna racemosa (Mill.) Irwin et Barneby 1

Ar. S. septemtrionalis (Viviani) H. S. Irwin et Barneby 1, 2

$\mathrm{H}$. Trifolium repens $\mathrm{L}$. 1

\section{LENTIBULARIACEAE}

H. Pinguicula moranensis Kunth 1

\section{LILIACEAE}

H. Echeandia mexicana Cruden 1

H. Smilacina paniculata M. Martens et Galeotti 2

Be. Smilax aristolochiifolia Mill. 1, 2

Be. S. bona-nox L. 2

Be. S. jalapensis Schltdl. 2

Be. S. moranensis M. Martens et Galeotti 1, 2

Be. S. subpubescens A. DC. 1

Be. S. tomentosa Kunth 1, 2

\section{LOGANIACEAE}

Ab. Buddleia cordata Kunth ssp. tometella (Standl.) Norman 1, 2

Ab. Buddleia sp. 1

\section{LORANTHACEAE}

P. Phoradendron velutinum (DC.) Nutt. 2

\section{LYTHRACEAE}

H. Cuphea aequipetala Cav. 1, 2 
Apéndice. Continuación.
H. C. calaminthifolia Schltdl. 2
H. C. cyanea DC. 1
H. C. procumbens Ortega 1,2
$\mathrm{H} . \quad$ Lythrum gracile Benth. 1

\section{MAGNOLIACEAE}

Aa. Magnolia schiedeana Schltdl. 1, 2

MALVACEAE

Ar. Malvaviscus arboreus Cav. 1

Ar. Phymosia umbellata (Cav.) Kearney 1

H. Sida rhombifolia L. 1,2

\section{MELASTOMATACEAE}

Ar. Leandra cornoides (Schltdl. et Cham.) Cogn. 1, 2

Ar. Miconia anisotricha (Schltdl.) Triana 2

Ar. M. oligotricha (DC.) Naudin 2

H. Tibouchina galeottiana Cogn. 2

H. T. mexicana (G. Don) Cogn. 1, 2

\section{MELIACEAE}

Ab. Trichilia havanensis Jacq. 2

\section{MORACEAE}

Ab. Trophis mexicana (Liebm.) Bureau 1

MYRSINACEAE

Ab. Ardisia compressa Kunth 1

Ar. Ardisia sp. 1

MYRTACEAE

Ar. Calycorectes mexicanus Berg 2

NYSSACEAE

Am. Nyssa sylvatica Marshall 2

\section{ONAGRACEAE}

Ar. Fuchsia arborescens Sims 2

Ar. F. microphylla Kunth ssp. hidalgensis (Munz) Breedlove 2

H. Lopezia racemosa Cav. var. racemosa 1, 2

H. Oenothera deserticola (Loesener) Munz 1

H. O. rosea L'Hér. 1, 2

H. O. tetraptera Cav. 1

\section{ORCHIDACEAE}

$\mathrm{H}$. Aulosepalum pyramidale (Lindl.) Soto Arenas 1

H. Calanthe calanthoides (A. Rich. et Galeotti) Hamer et Garay 2

H. $\quad$ Cranichis ciliilabia C. Schweinf. 2 
Apéndice. Continuación.

Ep. Domingoa kienastii (Rchb. f.) Dressler 2

Ep. Encyclia cyanocolumna (Ames, F. T. Hubb. et C. Schweinf.) Dressler 2

Ep. Epidendrum longipetalum A. Rich. et Galeotti 1, 2

H. Goodyera striata Rchb. f. 2

H. Govenia liliacea (La Llave et Lex.) Lindl. 1

Ep. Isochilus unilateralis Rob. 1

H. Malaxis aff. brachystachys (Rchb. f.) Kuntze 1

H. M. aff. histionantha (Link, Klotzsch et Otto) Garay et Dunst 1

Ep. M. majanthemifolia Schltdl. et Cham. 1

H. Ponthieva ephippium Rchb. f. 1

H. Prescottia stachyoides (Sw.) Lindl. 2

Ep. Rhynchostele rossii (Lindl.) Soto Arenas et Salazar 2

Ep. $\quad R$. aff. rossii (Lindl.) Soto Arenas et Salazar 1

H. Sarcoglottis schaffneri (Rchb. f.) Ames 1

OROBANCHACEAE

P. Conopholis alpina Liebm. 2

OXALIDACEAE

H. Oxalis alpina (Rose) Knuth 1

Ar. O. rhombifolia Jacq. 1

PALMAE

H. Chamaedorea elegans Mart. 1

PAPAVERACEAE

Ar. Bocconia frutescens L. 1

PASSIFLORACEAE

Be. Passiflora sicyoides Schltdl. et Cham. 1, 2

PHYTOLACCACEAE

H. Phytolacca purpurascens A. Braun et Bouché 1, 2

H. Rivina humilis L. 2

PIPERACEAE

Ep. Peperomia collocata Trel. 1,2

Ep. $\quad$ P. quadrifolia (L.) Kunth 1, 2

Ar. Piper amalago L. 1, 2

Ar. $\quad$ P. auritum Kunth 1, 2

Ar. $\quad$ P. fraguanum Trel. 1, 2

PLANTAGINACEAE

H. Plantago australis Lam. ssp. australis 1, 2

H. $\quad$ P. australis Lam. ssp. hirtella (Kunth) Rahan 1

POLEMONIACEAE

Be. Cobaea stipularis Benth. 1, 2 
Apéndice. Continuación.

POLYGALACEAE

Ar. Monnina xalapensis Kunth 1, 2

POLYGONACEAE

H. Polygonum punctatum Elliot 2

RANUNCULACEAE

Be. Clematis acapulcensis Hook. et Arn. 1, 2

$\mathrm{H}$. Ranunculus dichotomus Moc. et Sessé ex DC. 1

$\mathrm{H} . \quad$ R. geoides Kunth var. geoides 1

H. Thalictrum pubigerum Benth. 1, 2

RHAMNACEAE

Ar. Ceanothus coeruleus Lag. 1

Ab. Rhamnus capreaefolia Schltdl. 1, 2

Ab. R. longistyla C. B. Wolf 2

Ab. R. pompana M. C. Johnst. et L. A. Johnst. 1, 2

Ar. Rhamnus sp. 1

ROSACEAE

H. Acaena elongata L. 1, 2

$\mathrm{H}$. Alchemilla pectinata Kunth 1, 2

Am. Cercocarpus macrophyllus C. Schneid. 1

Ab. Crataegus mexicana Moc. et Sessé 1, 2

$\mathrm{H} . \quad$ Duchesnea indica (Andr.) Focke 2

Aa. Prunus brachybotrya Zucc. 1

Am. P. samydoides Schltdl. 1, 2

Ab. $\quad$ P. serotina Ehrb. ssp. capuli (Cav.) McVaugh 1, 2

Ar. Rubus adenotrichus Schltdl. 2

Ar. $\quad R$. schiedeanus Steud. 2

RUBIACEAE

H. Borreria laevis (Lam.) Griseb. 2

H. Bouvardia laevis M. Martens et Galeotti 1

H. B. xylosteoides Hook. et Arn. 1

H. Coccocypselum cordifolium Nees et Mart. 2

H. Crusea longiflora (Willd. ex Roem. et Schult.) W. R. Anderson 1, 2

Ar. Deppea microphylla Greenm. 1, 2

Ar. D. purpusii Standl. 1, 2

H. Galium uncinulatum DC. 1

H. Hoffmannia montana L. O. Williams 1, 2

H. Houstonia lanceolata (Poir.) Britton 2

H. Nertera granadensis (L.) Druce 2

Ar. Randia laetevirens Standl. 1, 2

Ar. Rondeletia capitellata Hemsl. 1, 2

RUTACEAE

Ab. Decatropis bicolor (Zucc.) Radlk. 1 
Apéndice. Continuación.

Ar. Peltostigma pteleoides (Hook.) Walp. 1

Am. Ptelea trifoliata L. 1

Am. Zanthoxylum clava-herculis L. 1, 2

Be. Z. foliolosum F. Donn. Sm. 2

Am. Z. xicense Miranda 1, 2

SABIACEAE

Aa. Meliosma alba (Schltdl.) Walp. 1

SAPOTACEAE

Am. Dipholis minutiflora Pittier 1

SAXIFRAGACEAE

$\mathrm{H}$. Heuchera orizabensis Hemsl. 1

Be. Philadelphus affinis Schltdl. 1, 2

Be. $\quad$ P. mexicanus Schltdl. 1, 2

\section{SCROPHULARIACEAE}

H. Calceolaria mexicana Benth. 1

H. Castilleja lithospermoides Kunth 1

H. C. tenuiflora Benth. 1

H. Digitalis purpurea L. 2

Be. Maurandya barclaiana Lindl. 1

\section{SIMAROUBACEAE}

Ab. Picramnia xalapensis Planch. 1

\section{SOLANACEAE}

$\mathrm{H}$. Athenaea viscosa (Schrad.) Fernald 2

$\mathrm{H} . \quad$ Capsicum sp. 1

Ar. Cestrum anagyris Dunal 2

Ar. C. elegans (Brongn.) Schltdl. 2

Ar. C. fasciculatum (Schltdl.) Miers 1, 2

Ar. C. nocturnum L. 1, 2

Ar. C. oblongifolium Schltdl. 1

Ar. Cyphomandra betacea (Cav.) Sendtn. 2

H. Physalis pubescens L. 1

$\mathrm{H}$. Physalis sp. 1

Ar. Solanum acerifolium Dunal 2

Ab. S. aligerum Schltdl. 1, 2

Ar. S. aphyodendron S. Knapp 1

Be. S. appendiculatum Dunal 1,2

H. S. diflorum Vell. 1

Ar. S. hispidum Pers. 1, 2

$\mathrm{H}$. S. morelliforme Bitter et Muench 2

H. S. myriacanthum Dunal 2

Ar. S. nigrescens M. Martens et Galeotti 1

Ar. S. nudum Dunal 1, 2 
Apéndice. Continuación.

Be. Solandra sp. 1

Ar. Witheringia stellata (Greenm.) Hunz. 1

STAPHYLEACEAE

Am. Turpinia occidentalis (Sw.) G. Don 1, 2

STYRACACEAE

Am. Styrax glabrescens Benth. 1, 2

SYMPLOCACEAE

Ab. Symplocos limoncillo Bonpl. 2

THEACEAE

Am. Cleyera theaeoides (Sw.) Choisy 1, 2

Ab. Ternstroemia huasteca B. M. Barthol. 2

Ab. T. sylvatica Schldlt. et Cham. 2

TILIACEAE

Ab. Heliocarpus appendiculatus Turcz. 2

Aa. Tilia houghi Rose 1

Ar. Triumfetta grandiflora Vahl 2

ULMACEAE

Ab. Lozanella enantiophylla (Donn. Sm.) Killip et C. V. Morton 1, 2

UMBELLIFERAE

H. Arracacia atropurpurea (Lehm.) Benth. et Hook. 1

H. Hydrocotyle mexicana Cham. et Schltdl. 2

H. Tauschia nudicaulis Schult. 1

\section{URTICACEAE}

Ar. Myriocarpa longipes Liebm. 1

H. $\quad$ Phenax hirtus (Sw.) Wedd. 1

H. $\quad$ P. mexicanus Wedd. 1

H. Phenax sp. 1

H. Pilea pubescens Liebm. 1

Ar. Urera baccifera (L.) Gaud. 1

\section{VALERIANACEAE}

Be. Valeriana candolleana Gardner 1

$\mathrm{H}$. V. clematitis Kunth 1

\section{VERBENACEAE}

Ab. Citharexylum hidalgense Moldenke 1, 2

Ar. Lantana hirta Graham 1

Ar. Lippia myriocephala Schltdl. et Cham. 1

H. Verbena elegans Kunth 1

$\mathrm{H}$. V. litoralis Kunth 2 
Alcántara y Luna: Análisis Florístico de dos Áreas con Bosque Mesófilo de Montaña Apéndice. Continuación.

VITACEAE

Be. Vitis popenoei J.H. Fennell 1, 2 